\title{
FINITE ELEMENT ANALYSIS OF SLOSHING AND HYDROELASTIC VIBRATIONS UNDER GRAVITY
}

\author{
Alfredo Bermúdez ${ }^{1}$ And Rodolfo Rodríguez ${ }^{2}$
}

\begin{abstract}
This paper deals with a finite element method to solve fluid-structure interaction problems. More precisely it concerns the numerical computation of harmonic hydroelastic vibrations under gravity. It is based on a displacement formulation for both the fluid and the solid. Gravity effects are included on the free surface of the fluid as well as on the liquid-solid interface. The pressure of the fluid is used as a variable for the theoretical analysis leading to a well posed mixed linear eigenvalue problem. Lowest order triangular Raviart-Thomas elements are used for the fluid and classical piecewise linear elements for the solid. Transmission conditions at the fluid-solid interface are taken into account in a weak sense yielding a non conforming discretization. The method does not present spurious or circulation modes for nonzero frequencies. Convergence is proved and optimal error estimates are given. Finally, numerical results are shown.

Résumé. Cet article concerne une méthode d'éléments finis pour la résolution de problèmes d'intéraction d'un fluide avec une structure. Plus précisement il s'agit de calculer les vibrations hydroélastiques harmoniques sous gravité. La méthode est basée sur une formulation en déplacements à la fois pour le solide et le fluide. Les effets de gravité sont inclus sur la surface libre du fluide et sur l'interphase entre le fluide et le solide. La pression dans le fluide est utilisée comme variable pour l'analyse théorique de la méthode ce qui conduit à un problème mixte aux valeurs propres bien posé. L'élément triangulaire de Raviart-Thomas du plus bas degré est utilisé pour discrétiser le fluide ; pour le solide on utilise des éléments finis linéaires par morceaux classiques. La condition de transmission cinématique à l'interphase est prise en compte de façon faible ce qui donne une discrétisation non conforme. La méthode ne produit pas des modes parasites rotationnels pour des fréquences non nulles. On démontre aussi la convergence et des estimations d'erreur qui sont optimales. Finalement, quelques résultats numériques sont présentés.
\end{abstract}

AMS Subject Classification. 65N25, 65N30, 70J10, 73K70.

Received: July 7, 1997. Revised: May 11, 1998.

Keywords and phrases. Fluid-structure, finite elements, hydroelasticity under gravity, vibrations, spectral problems, spurious modes.

1 Departamento de Matemática Aplicada, Universidade de Santiago de Compostela, 15706 Santiago de Compostela, Spain. Partially supported by Programa de Cooperación Científica con Iberoamérica, M.E.C. and Acción Integrada Hispano-Francesa HF 1997-0194, Spain.

2 Departamento de Ingeniería Matemática, Universidad de Concepción, Casilla 4009, Concepción, Chile. Partially supported by FONDECYT (Chile) through its grant No. 1.960 .615 and Subprogram A on Numerical Analysis of FONDAP in Applied Mathematics (Chile).

(C) EDP Sciences, SMAI 1999 


\section{INTRODUCTION}

Increasing attention has recently been paid to problems involving fluid-structure interactions. For a survey of current results see $[10,17]$ and the references therein. In this paper, we are concerned with hydroelastic vibrations under gravity. We consider as a model problem a 2D elastic vessel partially filled by an incompressible or nearly incompressible fluid (typically a liquid) with an open or free boundary. Gravity effects are considered by using the Tong model on the fluid-solid interface (see [21]) and a classical first order approximation of the kinetic condition on the free interface of the fluid.

Under the usual assumptions leading to linear problems, the evolution of the coupled system is governed by second order in time linear equations. Their solution can be written in terms of the corresponding free vibration modes which are eigenfunctions of a linear eigenvalue problem (see for instance [7]). The hydroelastic problem has been dealt with in previous papers by using different primal variables for fluid and solid: typically displacements for the structure, and velocity [19], velocity potential $[2,6]$ or displacement potential $[7,17]$ for the fluid. We have used displacement variables for both, fluid and solid; to provide a theoretical analysis, the pressure in the fluid has also been used as a variable.

As it is well known, spurious modes appear when a displacement formulation is discretized by using classical Lagrangian finite elements (see $[5,15])$. Such spurious modes are approximations of pure rotational motions of the fluid not inducing vibrations on the structure, which are zero frequency eigenmodes of the continuous problem. Therefore, when the discrete problem does not have zero as an eigenfrequency with a corresponding eigenspace approximating this set of rotational motions, spurious eigenmodes arise with non zero frequencies placed among those of the relevant ones.

In [3] a finite element method which does not present spurious modes is introduced for the case of a compressible fluid. It consists of using piecewise linear elements for the solid and Raviart-Thomas elements of lowest order for the fluid, the coupling of both being of non conforming type. Such discretization yields a sparse linear symmetric eigenvalue problem. In [4] it is shown that this method can be adapted to deal with incompressible fluids too.

In the present paper the previous results are extended to the case where gravity effects are taken into account: we give similar theorems concerning convergence and error estimates, and show that spurious modes do not arise, but using a different approach to that in the above mentioned references. Indeed, the approach in those papers could be extended to take into account the new terms in the variational formulation and the free boundary, however it would only allow to prove non optimal order error estimates. Instead, we present an alternative analysis leading to optimal orders of convergence. Furthermore, it allows to consider more complex geometries. In particular, the case of fluid domains with interior angles of $2 \pi$ is now covered. Thus the method can be used to simulate the effect of very thin baffle-plates which are included in some liquid reservoirs to avoid excessive sloshing.

Finally, we discuss implementation issues and present a numerical experiment showing the effectiveness of the method. We compute the sloshing and the elastic modes of an elastic vessel containing a liquid with a free boundary and estimate in both cases the orders of convergence.

\section{THE MODEL PROBLEM}

We consider the problem of determining the vibration modes of a linear elastic structure containing an inviscid fluid. Our model problem consists of a 2D polygonal vessel filled with a fluid with an open boundary as that in Figure 1.

Let $\Omega_{\mathrm{F}}$ and $\Omega_{\mathrm{S}}$ be the domains occupied by the fluid and the solid, respectively, which are not supposed to be convex or simply connected; even interior angles of $2 \pi$ are allowed. Let us denote by $\Gamma_{\mathrm{F}}$ the boundary of the fluid domain and by $\boldsymbol{\nu}$ its unit normal vector pointing outwards $\Omega_{\mathrm{F}}$. This boundary is split into two parts: the interface between the solid and the fluid $\Gamma_{\mathrm{I}}$ and the open boundary of the fluid $\Gamma_{\mathrm{O}}$. On the other hand, the solid boundary is the union of three parts: the interface $\Gamma_{\mathrm{I}}, \Gamma_{\mathrm{D}}$ and $\Gamma_{\mathrm{N}}$; the structure is supposed to be 


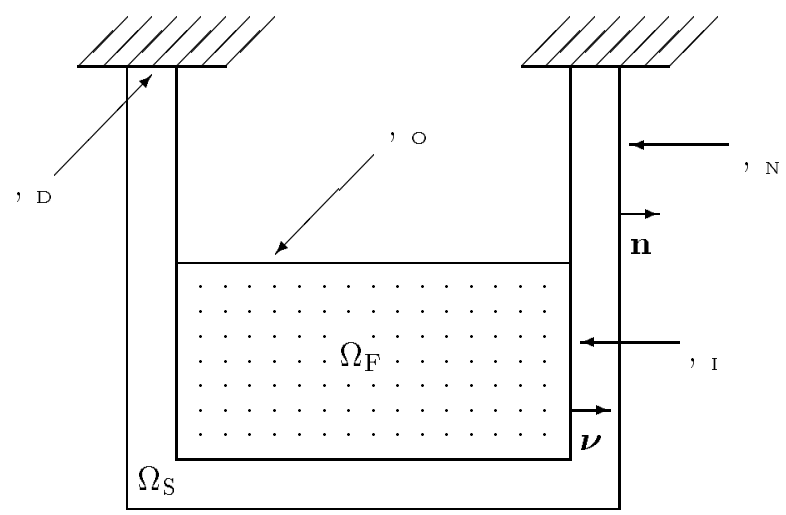

FiguRE 1. Fluid and solid domains.

fixed along $\Gamma_{\mathrm{D}}$ (meas $\Gamma_{\mathrm{D}}>0$ is assumed for simplicity) and free of stress along $\Gamma_{\mathrm{N}}$. Finally $\mathbf{n}$ denotes the unit outward normal vector along $\Gamma_{\mathrm{N}}$.

Throughout this paper we use the standard notation for Sobolev spaces, norms and seminorms. We also denote $H\left(\operatorname{div}, \Omega_{\mathrm{F}}\right):=\left\{\mathbf{u} \in L^{2}\left(\Omega_{\mathrm{F}}\right)^{2}: \operatorname{div} \mathbf{u} \in L^{2}\left(\Omega_{\mathrm{F}}\right)\right\}$ and $\|\mathbf{u}\|_{H\left(\operatorname{div}, \Omega_{\mathrm{F}}\right)}^{2}:=\|\mathbf{u}\|_{L^{2}\left(\Omega_{\mathrm{F}}\right)^{2}}^{2}+\|\operatorname{div} \mathbf{u}\|_{L^{2}\left(\Omega_{\mathrm{F}}\right)}^{2}$. We denote by $C$ a generic constant not necessarily the same at each occurrence.

We use the following notations for the physical magnitudes in the fluid:

$\mathbf{u}$ : the displacement vector,

$p$ : the pressure,

$\rho_{\mathrm{F}}$ : the density,

$c$ : the acoustic speed,

and in the solid:

$\mathbf{v}$ : the displacement vector, $\rho_{\mathrm{S}}:$ the density,

$\lambda_{\mathrm{S}}$ and $\mu_{\mathrm{S}}$ : the Lamé coefficients,

$\varepsilon(\mathbf{v})$ : the strain tensor defined by $\varepsilon_{i j}(\mathbf{v}):=\frac{1}{2}\left(\frac{\partial v_{i}}{\partial x_{j}}+\frac{\partial v_{j}}{\partial x_{i}}\right), i, j=1,2$,

$\boldsymbol{\sigma}(\mathbf{v})$ : the stress tensor, which we assume is related to the strains by Hooke's law:

$$
\sigma_{i j}(\mathbf{v})=\lambda_{\mathrm{s}} \sum_{k=1}^{2} \varepsilon_{k k}(\mathbf{v}) \delta_{i j}+2 \mu_{\mathrm{s}} \varepsilon_{i j}(\mathbf{v}), \quad i, j=1,2
$$

Gravity forces produce displacements in the solid $\mathbf{v}^{0}$ and prestresses $\sigma^{0}:=\boldsymbol{\sigma}\left(\mathbf{v}^{0}\right)$. We are interested in small amplitude motions departing from this prestressed equilibrium state. The classical linearization procedure yields the following approximate expression for the first Piola-Kirchoff stress tensor $\boldsymbol{\theta}$ (see Chapter 6 of [17] or [16]):

$$
\boldsymbol{\theta}=\boldsymbol{\sigma}^{0}+\nabla \mathbf{v} \boldsymbol{\sigma}^{0}+a_{i j k l} \varepsilon_{k l}(\mathbf{v})
$$

where $\mathbf{v}$ is the displacement field with respect to the prestressed equilibrium state.

In general, the second term on the right-hand side of the expression above may be neglected when compared with the third one. Indeed, $\boldsymbol{\sigma}^{0}$ is of the order of $\left(\rho_{\mathrm{S}} g L_{\mathrm{S}}+p^{0}\right)$, with $L_{\mathrm{S}}$ a typical length of the structure and $p^{0}$ the static pressure exerted by the fluid; this pressure is in its turn of the order of $\rho_{\mathrm{F}} g L_{\mathrm{F}}$, with $L_{\mathrm{F}}$ a typical depth of the fluid domain. In real problems the Lamé coefficients are very much larger than $\rho_{\mathrm{F}} g L_{\mathrm{F}}$ and $\rho_{\mathrm{S}} g L_{\mathrm{S}}$, allowing to neglect $\nabla \mathbf{v} \boldsymbol{\sigma}^{0}$, what will be done in the rest of the paper.

We notice that the term $\nabla \mathbf{v} \boldsymbol{\sigma}^{0}$ can be very important in some other situations, mostly related to slender or thin structures where reduced dimension models are used instead of the standard linear elasticity equations. 
Let us mention, for instance, the vibrations of an inflated rubber tire. However these cases are beyond the scope of the present paper and they will not be considered.

The classical linearization procedure yields the following eigenvalue problem for the vibration modes of the coupled system and their corresponding frequencies $\omega$ (see, for instance, [17]).

Find $\omega \geq 0, \mathbf{u}: \Omega_{\mathrm{F}} \rightarrow \mathbb{R}^{2}, \mathbf{v}: \Omega_{\mathrm{S}} \rightarrow \mathbb{R}^{2}$ and $p: \Omega_{\mathrm{F}} \rightarrow \mathbb{R},(\mathbf{u}, \mathbf{v}, p) \neq(\mathbf{0}, \mathbf{0}, 0)$, such that:

$$
\begin{aligned}
\nabla p-\omega^{2} \rho_{\mathrm{F}} \mathbf{u}=\mathbf{0}, & \text { in } \Omega_{\mathrm{F}}, \\
\frac{1}{\rho_{\mathrm{F}} c^{2}} p+\operatorname{div} \mathbf{u}=0, & \text { in } \Omega_{\mathrm{F}}, \\
\operatorname{div}[\boldsymbol{\sigma}(\mathbf{v})]+\omega^{2} \rho_{\mathrm{S}} \mathbf{v}=\mathbf{0}, & \text { in } \Omega_{\mathrm{S}}, \\
\mathbf{u} \cdot \boldsymbol{\nu}-\mathbf{v} \cdot \boldsymbol{\nu}=0, & \text { on } \Gamma_{\mathrm{I}}, \\
\boldsymbol{\sigma}(\mathbf{v}) \boldsymbol{\nu}+p \boldsymbol{\nu}=\rho_{\mathrm{F}} g \mathbf{k} \cdot \boldsymbol{\nu} \mathbf{v} \cdot \boldsymbol{\nu} \boldsymbol{\nu}, & \text { on } \Gamma_{\mathrm{I}}, \\
\rho_{\mathrm{F}} g \mathbf{u} \cdot \boldsymbol{\nu}-p=0, & \text { on } \Gamma_{\mathrm{O}}, \\
\boldsymbol{\sigma}(\mathbf{v}) \mathbf{n}=\mathbf{0}, & \text { on } \Gamma_{\mathrm{N}}, \\
\mathbf{v}=\mathbf{0}, & \text { on } \Gamma_{\mathrm{D}} .
\end{aligned}
$$

The coupling between the fluid and the structure is taken into account by equations (2.4) and (2.5) (in the latter $\mathbf{k}:=(0,1)$ denotes the unit vertical vector). The first one means that fluid and solid are in contact at the interface. The second one relates normal stresses of the solid on the interface with the pressure into the fluid and gravity effects. Following Tong [21], the latter are modelled by the term in the right hand side of (2.5).

The problem with a perfectly incompressible fluid can be thought of as the limit case of the previous one as $c$ goes to infinity. In this case (2.2) could be replaced by the simpler condition div $\mathbf{u}=0$. In order to deal with both cases in a same framework we consider $\frac{1}{\rho_{\mathrm{F}} c^{2}}=0$ for an incompressible fluid (i.e., $\left.c=\infty\right)$. Thus $(2.2)$ also makes sense in this case. All what follows in this paper is valid for $c=\infty$ as well as for finite values of $c$.

\section{VARIATIONAL FORMUlation}

A similar problem was considered in [4], but for a closed vessel completely filled with fluid and neglecting the gravity effects. In this section we extend the results in that reference to cover our problem. To this goal, we introduce the functional spaces $Q:=L^{2}\left(\Omega_{\mathrm{F}}\right), \mathbf{H}:=L^{2}\left(\Omega_{\mathrm{F}}\right)^{2} \times L^{2}\left(\Omega_{\mathrm{S}}\right)^{2}$ and

$$
\mathbf{X}:=\left\{(\mathbf{u}, \mathbf{v}) \in H\left(\operatorname{div}, \Omega_{\mathrm{F}}\right) \times H_{\Gamma_{\mathrm{D}}}^{1}\left(\Omega_{\mathrm{S}}\right)^{2}: \mathbf{u} \cdot \boldsymbol{\nu} \in L^{2}\left(\Gamma_{\mathrm{F}}\right)\right\}
$$

where $H_{\Gamma_{\mathrm{D}}}^{1}\left(\Omega_{\mathrm{S}}\right)$ is the subspace of functions in $H^{1}\left(\Omega_{\mathrm{S}}\right)$ vanishing on $\Gamma_{\mathrm{D}}$. We denote by $\|\cdot\|$ the natural norm on $\mathbf{X}$ :

$$
\|(\mathbf{u}, \mathbf{v})\|:=\left[\|\mathbf{u}\|_{H\left(\operatorname{div}, \Omega_{\mathrm{F}}\right)}^{2}+\|\mathbf{u} \cdot \boldsymbol{\nu}\|_{L^{2}\left(\Gamma_{\mathrm{F}}\right)}^{2}+\|\mathbf{v}\|_{H^{1}\left(\Omega_{\mathrm{S}}\right)^{2}}^{2}\right]^{1 / 2}
$$

Let $\mathbf{V}$ be defined by

$$
\mathbf{V}:=\left\{(\mathbf{u}, \mathbf{v}) \in \mathbf{X}: \mathbf{u} \cdot \boldsymbol{\nu}=\mathbf{v} \cdot \boldsymbol{\nu}, \text { on } \Gamma_{\mathrm{I}}\right\}
$$

$\mathbf{V}$ is a closed subspace of $\mathbf{X}$ and, in this space, the norm (3.2) is equivalent to

$$
\left[\|\mathbf{u}\|_{H\left(\operatorname{div}, \Omega_{\mathrm{F}}\right)}^{2}+\|\mathbf{u} \cdot \boldsymbol{\nu}\|_{L^{2}\left(\Gamma_{\mathrm{O}}\right)}^{2}+\|\mathbf{v}\|_{H^{1}\left(\Omega_{\mathrm{S}}\right)^{2}}^{2}\right]^{1 / 2} .
$$

Finally, we denote by $|\cdot|$ the $L^{2}$ norm on $\mathbf{H}$ or on $Q$, as corresponds. 
It is easy to check that the following is a mixed variational formulation of the eigenvalue problem (2.1-2.8): Find $\lambda \in \mathbb{R},(\mathbf{u}, \mathbf{v}, p) \in \mathbf{V} \times Q,(\mathbf{u}, \mathbf{v}, p) \neq(\mathbf{0}, \mathbf{0}, 0)$, such that:

$$
\begin{gathered}
\int_{\Omega_{\mathrm{S}}} \boldsymbol{\sigma}(\mathbf{v}): \boldsymbol{\varepsilon}(\boldsymbol{\psi})+\int_{\Gamma_{\mathrm{O}}} \rho_{\mathrm{F}} g \mathbf{u} \cdot \boldsymbol{\nu} \boldsymbol{\phi} \cdot \boldsymbol{\nu} d \Gamma+\int_{\Gamma_{\mathrm{I}}} \rho_{\mathrm{F}} g \mathbf{k} \cdot \boldsymbol{\nu} \mathbf{v} \cdot \boldsymbol{\nu} \boldsymbol{\psi} \cdot \boldsymbol{\nu} d \Gamma-\int_{\Omega_{\mathrm{F}}} p \operatorname{div} \boldsymbol{\phi} \\
=\lambda\left(\int_{\Omega_{\mathrm{F}}} \rho_{\mathrm{F}} \mathbf{u} \cdot \boldsymbol{\phi}+\int_{\Omega_{\mathrm{S}}} \rho_{\mathrm{S}} \mathbf{v} \cdot \boldsymbol{\psi}\right), \quad \forall(\boldsymbol{\phi}, \boldsymbol{\psi}) \in \mathbf{V}, \\
\int_{\Omega_{\mathrm{F}}} q \operatorname{div} \mathbf{u}+\frac{1}{\rho_{\mathrm{F}} c^{2}} \int_{\Omega_{\mathrm{F}}} p q=0, \quad \forall q \in Q
\end{gathered}
$$

where $\lambda=\omega^{2}$ and $\boldsymbol{\sigma}(\mathbf{v}): \boldsymbol{\varepsilon}(\boldsymbol{\psi}):=\sum_{i, j=1,2} \sigma_{i j}(\mathbf{v}) \varepsilon_{i j}(\boldsymbol{\psi})$ denotes the usual inner product in the space of second order tensors.

From now on, we make the following assumption which is always fulfilled in real problems, as it is shown in the remark below:

Assumption 1. There exists a positive constant $\alpha$ such that

$$
\int_{\Omega_{\mathrm{S}}} \boldsymbol{\sigma}(\mathbf{v}): \boldsymbol{\varepsilon}(\mathbf{v})+\int_{\Gamma_{\mathrm{I}}} \rho_{\mathrm{F}} g \mathbf{k} \cdot \boldsymbol{\nu}(\mathbf{v} \cdot \boldsymbol{\nu})^{2} d \Gamma \geq \alpha \int_{\Omega_{\mathrm{S}}}|\nabla \mathbf{v}|^{2}, \quad \forall \mathbf{v} \in H_{\Gamma_{\mathrm{D}}}^{1}\left(\Omega_{\mathrm{S}}\right) .
$$

Remark 3.1. The previous assumption is readily satisfied in real situations. Indeed, from Korn's inequality we have that

$$
\int_{\Omega_{\mathrm{S}}} \boldsymbol{\sigma}(\mathbf{v}): \varepsilon(\mathbf{v}) \geq \gamma \int_{\Omega_{\mathrm{S}}}|\nabla \mathbf{v}|^{2}, \quad \forall \mathbf{v} \in H_{\Gamma_{\mathrm{D}}}^{1}\left(\Omega_{\mathrm{S}}\right)^{2}
$$

with a positive constant $\gamma$ of the order of the Lamé coefficients $\lambda_{\mathrm{S}}$ and $\mu_{\mathrm{S}}$. On the other hand,

$$
\left|\int_{\Gamma_{\mathrm{I}}} \rho_{\mathrm{F}} g \mathbf{k} \cdot \boldsymbol{\nu}(\mathbf{v} \cdot \boldsymbol{\nu})^{2} d \Gamma\right| \leq \rho_{\mathrm{F}} g C \int_{\Omega_{\mathrm{S}}}|\nabla \mathbf{v}|^{2}, \quad \forall \mathbf{v} \in H_{\Gamma_{\mathrm{D}}}^{1}\left(\Omega_{\mathrm{S}}\right)^{2},
$$

with a constant $C$ coming from the Trace Theorem and Poincaré's inequality; this constant is of the order of a typical length $L$ of the solid domain. In real problems, the Lamé coefficients are very much larger than $\rho_{\mathrm{S}} g L$ allowing (3.6) to hold.

Let us now consider the following continuous bilinear forms:

$$
\begin{aligned}
d((\mathbf{u}, \mathbf{v}),(\boldsymbol{\phi}, \boldsymbol{\psi})):= & \int_{\Omega_{\mathrm{F}}} \rho_{\mathrm{F}} \mathbf{u} \cdot \boldsymbol{\phi}+\int_{\Omega_{\mathrm{S}}} \rho_{\mathrm{S}} \mathbf{v} \cdot \boldsymbol{\psi}, \quad(\mathbf{u}, \mathbf{v}),(\boldsymbol{\phi}, \boldsymbol{\psi}) \in \mathbf{H}, \\
a((\mathbf{u}, \mathbf{v}),(\boldsymbol{\phi}, \boldsymbol{\psi})):= & \int_{\Omega_{\mathrm{S}}} \boldsymbol{\sigma}(\mathbf{v}): \boldsymbol{\varepsilon}(\boldsymbol{\psi})+\int_{\Gamma_{\mathrm{O}}} \rho_{\mathrm{F}} g \mathbf{u} \cdot \boldsymbol{\nu} \boldsymbol{\phi} \cdot \boldsymbol{\nu} d \Gamma \\
& +\int_{\Gamma_{\mathrm{I}}} \rho_{\mathrm{F}} g \mathbf{k} \cdot \boldsymbol{\nu} \mathbf{v} \cdot \boldsymbol{\nu} \boldsymbol{\psi} \cdot \boldsymbol{\nu} d \Gamma+d((\mathbf{u}, \mathbf{v}),(\boldsymbol{\phi}, \boldsymbol{\psi})), \quad(\mathbf{u}, \mathbf{v}),(\boldsymbol{\phi}, \boldsymbol{\psi}) \in \mathbf{X}, \\
b((\mathbf{u}, \mathbf{v}), q):= & -\int_{\Omega_{\mathrm{F}}} q \operatorname{div} \mathbf{u}, \quad(\mathbf{u}, \mathbf{v}) \in \mathbf{X}, \quad q \in Q,
\end{aligned}
$$

and the subspace of $\mathbf{V}$

$$
\mathbf{W}:=\{(\mathbf{u}, \mathbf{v}) \in \mathbf{V}: b((\mathbf{u}, \mathbf{v}), q)=0, \forall q \in Q\}=\{(\mathbf{u}, \mathbf{v}) \in \mathbf{V}: \operatorname{div} \mathbf{u}=0\}
$$


The following lemma shows that the bilinear forms $a$ and $b$ satisfy both classical Brezzi's conditions:

Lemma 3.1. The bilinear forms $a$ and $b$ satisfy:

i) a is coercive on $\mathbf{W}$;

ii) there exists a strictly positive constant $\beta$ such that

$$
\inf _{\substack{q \in Q \\ q \neq 0}} \sup _{\substack{(\mathbf{u}, \mathbf{v}) \in \mathbf{V} \\(\mathbf{u}, \mathbf{v}) \neq(\mathbf{0}, \mathbf{0})}} \frac{b((\mathbf{u}, \mathbf{v}), q)}{\|(\mathbf{u}, \mathbf{v})\||q|} \geq \beta .
$$

Proof. The coerciveness of $a$ in $\mathbf{W}$ is an immediate consequence of Assumption 1 and the fact that the norm $\|\cdot\|$ in $\mathbf{V}$ is equivalent to that in (3.3).

On the other hand, to prove the inf-sup condition (ii) it is enough to show that, for all $q \in Q=L^{2}\left(\Omega_{\mathrm{F}}\right)$, there exists $(\mathbf{u}, \mathbf{v}) \in \mathbf{V}$ satisfying

$$
\operatorname{div} \mathbf{u}=q \quad \text { in } \Omega_{\mathrm{F}} \quad \text { and } \quad\|(\mathbf{u}, \mathbf{v})\| \leq C|q| .
$$

This can be shown by adapting the proof of Lemma 3.1 in [4] to our case. In fact, let $\Omega:=\left(\bar{\Omega}_{\mathrm{S}} \cup \bar{\Omega}_{\mathrm{F}}\right)^{\circ}$; let $\tilde{q} \in L^{2}(\Omega)$ be the extension of $q$ obtained by defining

$$
\tilde{q}:=-\frac{1}{\left|\Omega_{\mathrm{S}}\right|} \int_{\Omega_{\mathrm{F}}} q \quad \text { in } \Omega_{\mathrm{S}} .
$$

Therefore, $\tilde{q} \in L_{0}^{2}(\Omega):=\left\{q \in L^{2}(\Omega): \int_{\Omega} q=0\right\}$. Since div is an isomorphism of a subspace of $\left[H_{0}^{1}(\Omega)\right]^{2}$ onto $L_{0}^{2}(\Omega)$ (see [12]), then there exists $\mathbf{w} \in\left[H_{0}^{1}(\Omega)\right]^{2}$ such that

$$
\operatorname{div} \mathbf{w}=\tilde{q} \quad \text { in } \Omega \quad \text { and } \quad\|\mathbf{w}\|_{\left[H^{1}(\Omega)\right]^{2}} \leq C\|\tilde{q}\|_{L^{2}(\Omega)},
$$

with $C$ independent of $q$. Let $\mathbf{u}:=\left.\mathbf{w}\right|_{\Omega_{\mathrm{F}}}$ and $\mathbf{v}:=\left.\mathbf{w}\right|_{\Omega_{\mathrm{S}}} ;$ hence, $(\mathbf{u}, \mathbf{v}) \in \mathbf{V}$ and, since $\mathbf{u} \cdot \boldsymbol{\nu}=0$ on $\Gamma_{\mathrm{O}}$, it clearly satisfies (3.7).

As a consequence of this lemma (see, for instance, [8]), given $(\mathbf{f}, \mathbf{g}) \in \mathbf{H}$, there exists a unique solution $(\mathbf{u}, \mathbf{v}, p) \in \mathbf{V} \times Q$ of the mixed source problem

$$
\begin{array}{clrl}
a((\mathbf{u}, \mathbf{v}),(\boldsymbol{\phi}, \boldsymbol{\psi}))+b((\boldsymbol{\phi}, \boldsymbol{\psi}), p) & =d((\mathbf{f}, \mathbf{g}),(\boldsymbol{\phi}, \boldsymbol{\psi})), & & \forall(\boldsymbol{\phi}, \boldsymbol{\psi}) \in \mathbf{V} \\
b((\mathbf{u}, \mathbf{v}), q)-\frac{1}{\rho_{\mathrm{F}} c^{2}} \int_{\Omega_{\mathrm{F}}} p q=0, & & \forall q \in Q
\end{array}
$$

and, moreover,

$$
\|(\mathbf{u}, \mathbf{v})\|+|p| \leq C|(\mathbf{f}, \mathbf{g})|,
$$

with a constant $C$ independent of the acoustic speed $c$ (even for $c=\infty$ ).

Let us denote by $\mathbf{T}$ the operator defined by

$$
\mathbf{T}: \begin{array}{cl}
\mathbf{H} & \longrightarrow \mathbf{V} \subset \mathbf{H} \\
(\mathbf{f}, \mathbf{g}) & \longmapsto(\mathbf{u}, \mathbf{v})
\end{array}
$$

with $(\mathbf{u}, \mathbf{v}, p)$ being the solution of (3.9-3.10); because of (3.11), $\mathbf{T}$ is a bounded linear operator. Since the bilinear forms $a$ and $d$ are symmetric, $\mathbf{T}$ is self-adjoint with respect to $d$. Hence all of its eigenvalues are real and it is easily checked that they are non negative. 
On the other hand, $(\lambda,(\mathbf{u}, \mathbf{v}))$ is an eigenpair of $\mathbf{T}$ if and only if there exists $p \in L^{2}\left(\Omega_{\mathrm{F}}\right)$ such that $\left(\frac{1}{\lambda}-1,(\mathbf{u}, \mathbf{v}, p)\right)$ is a solution of (3.4-3.5). Therefore, the knowledge of the spectrum of $\mathbf{T}$ gives complete information about the solutions of our original problem.

\section{Characterization of the spectrum and A Priori estimates}

Given any function $\mathbf{u} \in H\left(\operatorname{div}, \Omega_{\mathrm{F}}\right)$ with $\operatorname{div} \mathbf{u}=0$ and $\mathbf{u} \cdot \boldsymbol{\nu}=0,(\mathbf{u}, \mathbf{0}, 0) \in \mathbf{V} \times Q$ is an eigenfunction of problem (3.4)-(3.5) associated with the eigenvalue $\lambda=0$. Equivalently, $\lambda=1$ is an eigenvalue of $\mathbf{T}$ and it is shown below that

$$
\mathbf{K}:=\left\{(\mathbf{u}, \mathbf{0}): \mathbf{u} \in H\left(\operatorname{div}, \Omega_{\mathrm{F}}\right), \operatorname{div} \mathbf{u}=0 \text { in } \Omega_{\mathrm{F}} \text { and } \mathbf{u} \cdot \boldsymbol{\nu}=0 \text { on } \Gamma_{\mathrm{F}}\right\}
$$

is the corresponding eigenspace. The following characterization (see [12]),

$$
\mathbf{K}=\left\{(\operatorname{curl} \xi, \mathbf{0}): \xi \in H^{1}\left(\Omega_{\mathrm{F}}\right) \text { and } \xi \text { is constant on each connected component of } \Gamma_{\mathrm{F}}\right\},
$$

shows that this eigenspace consists of rotational motions of the fluid inducing neither vibrations in the solid nor variations of pressure.

Theorem 4.1. $\lambda=1$ is an eigenvalue of $\mathbf{T}$ with eigenspace $\mathbf{K}$.

Proof. It is a simple variation of that of Theorem 3.1 in [4]. In fact, for all $(\mathbf{u}, \mathbf{0}) \in \mathbf{K}$, clearly $\mathbf{T}(\mathbf{u}, \mathbf{0})=(\mathbf{u}, \mathbf{0})$. Conversely, let $(\mathbf{u}, \mathbf{v}) \in \mathbf{V}$ such that $\mathbf{T}(\mathbf{u}, \mathbf{v})=(\mathbf{u}, \mathbf{v})$; then,

$$
\begin{aligned}
& \int_{\Omega_{\mathrm{S}}} \boldsymbol{\sigma}(\mathbf{v}): \boldsymbol{\varepsilon}(\boldsymbol{\psi})+\int_{\Gamma_{\mathrm{O}}} \rho_{\mathrm{F}} g \mathbf{u} \cdot \boldsymbol{\nu} \boldsymbol{\phi} \cdot \boldsymbol{\nu} d \Gamma+\int_{\Gamma_{\mathrm{I}}} \rho_{\mathrm{F}} g \mathbf{k} \cdot \boldsymbol{\nu} \mathbf{v} \cdot \boldsymbol{\nu} \boldsymbol{\psi} \cdot \boldsymbol{\nu} d \Gamma-\int_{\Omega_{\mathrm{F}}} p \operatorname{div} \boldsymbol{\phi}=0, \quad \forall(\boldsymbol{\phi}, \boldsymbol{\psi}) \in \mathbf{V}, \\
& -\int_{\Omega_{\mathrm{F}}} q \operatorname{div} \mathbf{u}-\frac{1}{\rho_{\mathrm{F}} c^{2}} \int_{\Omega_{\mathrm{F}}} p q=0, \quad \forall q \in Q .
\end{aligned}
$$

Hence, $\operatorname{div} \mathbf{u}=-\frac{1}{\rho_{\mathrm{F}} c^{2}} p$ in $\Omega_{\mathrm{F}}$ and then, by using $(\phi, \psi)=(\mathbf{u}, \mathbf{v})$ in the first equation,

$$
\int_{\Omega_{\mathrm{S}}} \boldsymbol{\sigma}(\mathbf{v}): \varepsilon(\mathbf{v})+\int_{\Gamma_{\mathrm{O}}} \rho_{\mathrm{F}} g(\mathbf{u} \cdot \boldsymbol{\nu})^{2} d \Gamma+\int_{\Gamma_{\mathrm{I}}} \rho_{\mathrm{F}} g \mathbf{k} \cdot \boldsymbol{\nu}(\mathbf{v} \cdot \boldsymbol{\nu})^{2} d \Gamma+\frac{1}{\rho_{\mathrm{F}} c^{2}} \int_{\Omega_{\mathrm{F}}} p^{2}=0
$$

Therefore, because of Assumption 1, and the fact that $\mathbf{v}$ vanishes on $\Gamma_{\mathrm{D}}$, we have that $\mathbf{v}=\mathbf{0}$ in $\Omega_{\mathrm{S}}$. Moreover, $\mathbf{u} \cdot \boldsymbol{\nu}=0$ on $\Gamma_{\mathrm{F}}$ and, in the compressible case, $p=0$. Then, in both cases, $\mathbf{u}$ satisfies $\operatorname{div} \mathbf{u}=0$ in $\Omega_{\mathrm{F}}$ and $\mathbf{u} \cdot \boldsymbol{\nu}=0$ on $\Gamma_{\mathrm{F}}$, and hence $(\mathbf{u}, \mathbf{v}) \in \mathbf{K}$.

Because of the previous theorem, $\mathbf{T}$ is the identity on the infinite dimensional subspace $\mathbf{K}$; therefore $\mathbf{T}$ is not compact. However, as we show below, the restriction of $\mathbf{T}$ to the orthogonal complement of $\mathbf{K}$ is compact and this can be used to characterize the spectrum of $\mathbf{T}$.

The orthogonal complement of $\mathbf{K}$ in $\mathbf{H}$ is given by (see [12]):

$$
\mathbf{K}^{\perp_{\mathrm{H}}}=\left\{(\nabla \varphi, \mathbf{v}) \in \mathbf{H}: \varphi \in H^{1}\left(\Omega_{\mathrm{F}}\right), \mathbf{v} \in L^{2}\left(\Omega_{\mathrm{S}}\right)^{2}\right\} .
$$

Since $\rho_{\mathrm{F}}$ is constant on $\Omega_{\mathrm{F}}, \mathbf{K}$ and $\mathbf{K}^{\perp_{\mathrm{H}}}$ are also orthogonal with respect to the bilinear form $d$. Now $\mathbf{K} \subset \mathbf{V}$; let $\mathbf{G}$ denote the orthogonal complement of $\mathbf{K}$ in $\mathbf{V}$. It is easy to check that $\mathbf{G}:=\mathbf{K}^{\perp_{\mathrm{V}}}=\mathbf{K}^{\perp_{\mathrm{H}}} \cap \mathbf{V}$ and that $\mathbf{K}$ and $\mathbf{G}$ are also orthogonal with respect to $d$. Hence we have the following lemma and, as a consequence of it, $\mathbf{G}$ is an invariant subspace for $\mathbf{T}$.

Lemma 4.1. It holds that $\mathbf{T}\left(\mathbf{K}^{\perp}{ }_{\mathrm{H}}\right) \subset \mathbf{G}$. 
Proof. Since by Theorem 4.1, $\mathbf{K}$ is an invariant subspace for $\mathbf{T}$, the lemma is an immediate consequence of the orthogonality of $\mathbf{K}$ and $\mathbf{K}^{\perp_{H}}$ with respect to $d$ and the self-adjointness of $\mathbf{T}$ with respect to this inner product.

On the other hand, we have the following a priori estimate for $\mathbf{T}\left(\mathbf{K}^{\perp_{\mathrm{H}}}\right)$ :

Lemma 4.2. There exist constants $s \in\left[\frac{1}{2}, 1\right]$ and $C>0$ (not depending on $c$ ) such that if $(\mathbf{u}, \mathbf{v}, p) \in \mathbf{V} \times Q$ is the solution of problem (3.9)-(3.10) with $(\mathbf{f}, \mathbf{g}) \in \mathbf{K}^{\perp_{\mathrm{H}}}$, then $\mathbf{u} \in H^{s}\left(\Omega_{\mathrm{F}}\right)^{2}, \mathbf{u} \cdot \boldsymbol{\nu} \in H^{1 / 2}\left(\Gamma_{\mathrm{O}}\right), p \in H^{1}\left(\Omega_{\mathrm{F}}\right)$ and

$$
\|\mathbf{u}\|_{H^{s}\left(\Omega_{\mathrm{F}}\right)^{2}}+\|\mathbf{u} \cdot \boldsymbol{\nu}\|_{H^{1 / 2}\left(\Gamma_{\mathrm{O}}\right)}+\|p\|_{H^{1}\left(\Omega_{\mathrm{F}}\right)} \leq C|(\mathbf{f}, \mathbf{g})| .
$$

Proof. Let $(\mathbf{f}, \mathbf{g}) \in \mathbf{K}^{\perp_{\mathrm{H}}}$ and let $(\mathbf{u}, \mathbf{v}, p) \in \mathbf{V} \times Q$ be the solution of problem (3.9-3.10). By using $\phi \in \mathcal{C}_{0}^{\infty}\left(\Omega_{\mathrm{F}}\right)^{2}$ and $\boldsymbol{\psi}=\mathbf{0}$ in (3.9), it turns out that

$$
\nabla p+\rho_{\mathrm{F}} \mathbf{u}=\rho_{\mathrm{F}} \mathbf{f}
$$

Hence $p \in H^{1}\left(\Omega_{\mathrm{F}}\right)$ and, because of $(3.11)$,

$$
\|p\|_{H^{1}\left(\Omega_{\mathrm{F}}\right)} \leq C|(\mathbf{f}, \mathbf{g})| .
$$

On the other hand, by using $\phi \in \mathcal{C}^{\infty}\left(\bar{\Omega}_{\mathrm{F}}\right)^{2}$ such that $\operatorname{supp}(\phi) \cap \Gamma_{\mathrm{I}}=\emptyset$ and $\boldsymbol{\psi}=\mathbf{0}$, integrating by parts and using (4.1), we obtain

$$
\int_{\Gamma_{\mathrm{O}}} \rho_{\mathrm{F}} g \mathbf{u} \cdot \boldsymbol{\nu} \phi \cdot \boldsymbol{\nu} d \Gamma=\int_{\Gamma_{\mathrm{O}}} p \boldsymbol{\phi} \cdot \boldsymbol{\nu} d \Gamma
$$

Hence, $\rho_{\mathrm{F}} g \mathbf{u} \cdot \boldsymbol{\nu}=p$ on $\Gamma_{\mathrm{O}}$ and so

$$
\|\mathbf{u} \cdot \boldsymbol{\nu}\|_{H^{1 / 2}\left(\Gamma_{\mathrm{O}}\right)} \leq \frac{1}{\rho_{\mathrm{F}} g}\|p\|_{H^{1}\left(\Omega_{\mathrm{F}}\right)} \leq C|(\mathbf{f}, \mathbf{g})| .
$$

Now, because of Lemma 4.1, (u, v) $\in \mathbf{G}$ and then there exists $\varphi \in H^{1}\left(\Omega_{\mathrm{F}}\right)$ such that $\mathbf{u}=\nabla \varphi$. Since $\mathbf{u} \cdot \boldsymbol{\nu}=\mathbf{v} \cdot \boldsymbol{\nu}$ on $\Gamma_{\mathrm{I}}$ and, because of $(3.10), \operatorname{div} \mathbf{u}=-\frac{1}{\rho_{\mathrm{F}} c^{2}} p$, then $\varphi$ is a solution of the compatible Neumann problem:

$$
\begin{aligned}
-\Delta \varphi & =\frac{1}{\rho_{\mathrm{F}} c^{2}} p, & & \text { in } \Omega_{\mathrm{F}}, \\
\frac{\partial \varphi}{\partial \boldsymbol{\nu}} & =\frac{1}{\rho_{\mathrm{F}} g} p, & & \text { on } \Gamma_{\mathrm{O}}, \\
\frac{\partial \varphi}{\partial \boldsymbol{\nu}} & =\mathbf{v} \cdot \boldsymbol{\nu}, & & \text { on } \Gamma_{\mathrm{I}} .
\end{aligned}
$$

By using the standard a priori estimate for this Neumann problem (see, for instance, [13]) we know that $\varphi \in H^{1+s}\left(\Omega_{\mathrm{F}}\right)$, where $s=1$ if $\Omega_{\mathrm{F}}$ is convex, and $s=\pi / \theta$ ( $\theta$ being the biggest reentrant corner of $\left.\Omega_{\mathrm{F}}\right)$ otherwise; moreover,

$$
\|\mathbf{u}\|_{H^{s}\left(\Omega_{\mathrm{F}}\right)^{2}}=\|\nabla \varphi\|_{H^{s}\left(\Omega_{\mathrm{F}}\right)^{2}} \leq C\left[\sum_{j=1}^{J}\|\mathbf{v} \cdot \boldsymbol{\nu}\|_{H^{1 / 2}\left(\Gamma_{j}\right)}+\frac{1}{\rho_{\mathrm{F}} g}\|p\|_{H^{1 / 2}\left(\Gamma_{\mathrm{O}}\right)}+\frac{1}{\rho_{\mathrm{F}} c^{2}}\|p\|_{L^{2}\left(\Omega_{\mathrm{F}}\right)}\right] \leq C|(\mathbf{f}, \mathbf{g})|,
$$

where we have used (3.11) and (4.2) for the last inequality $\left(\Gamma_{j}, 1 \leq j \leq J\right.$, denote the edges of the polygonal interface $\Gamma_{\mathrm{I}}$ ). Notice that the last constant $C$ can be chosen independently of the value of $c$, for $c$ bounded below away from zero. Thus, (4.2), (4.3) and (4.4) allow us to conclude the lemma.

Now we can give a complete characterization of the eigenpairs of $\mathbf{T}$ and hence of the solutions of (3.4-3.5). 
Theorem 4.2. Except for $\lambda=0$, the spectrum of $\mathbf{T}$ consists of the eigenvalue $\lambda=1$ and a sequence of finite multiplicity eigenvalues $\left\{\lambda_{n}: n \in \mathbb{N}\right\} \subset(0,1)$ converging to $0 . \mathbf{K}$ is the eigenspace of $\lambda=1$ and each eigenfunction $\left(\mathbf{u}_{n}, \mathbf{v}_{n}\right)$ associated with an eigenvalue $\lambda_{n} \in(0,1)$ satisfies curl $\mathbf{u}_{n}=0$.

Proof. It is an immediate consequence of Theorem 4.1, Lemmas 4.1 and 4.2 and the fact that, for $s>0$, $H^{s}\left(\Omega_{\mathrm{F}}\right)^{2} \times H^{1}\left(\Omega_{\mathrm{S}}\right)^{2}$ is compactly embedded in $\mathbf{H}$.

In order to prove error estimates for the discretization to be introduced in the next section, further regularity of the eigenfunctions associated with $\lambda_{n} \in(0,1)$ is needed. Lemma 4.2 provides such regularity for the fluid displacements and the following Lemma for the solid ones.

Lemma 4.3. There exist constants $t \in(0,1]$ and $C>0$ such that if $(\mathbf{u}, \mathbf{v}, p) \in \mathbf{V} \times Q$ is the solution of problem (3.9)-(3.10) with $(\mathbf{f}, \mathbf{g}) \in \mathbf{K}^{\perp_{\mathrm{H}}}$, then $\mathbf{v} \in H^{1+t}\left(\Omega_{\mathrm{S}}\right)^{2}$ and

$$
\|\mathbf{v}\|_{H^{1+t}\left(\Omega_{\mathrm{S}}\right)^{2}} \leq C|(\mathbf{f}, \mathbf{g})| .
$$

Proof. For any $\boldsymbol{\psi} \in H_{\Gamma_{\mathrm{D}}}^{1}\left(\Omega_{\mathrm{S}}\right)^{2}$ let $\boldsymbol{\phi} \in H\left(\operatorname{div}, \Omega_{\mathrm{F}}\right)$ be such that $(\boldsymbol{\phi}, \boldsymbol{\psi}) \in \mathbf{V}$. Then, by using (3.9) and (4.1), we obtain

$$
\int_{\Omega_{\mathrm{S}}} \boldsymbol{\sigma}(\mathbf{v}): \boldsymbol{\varepsilon}(\boldsymbol{\psi})+\int_{\Gamma_{\mathrm{I}}} \rho_{\mathrm{F}} g \mathbf{k} \cdot \boldsymbol{\nu} \mathbf{v} \cdot \boldsymbol{\nu} \boldsymbol{\psi} \cdot \boldsymbol{\nu} d \Gamma+\int_{\Omega_{\mathrm{S}}} \rho_{\mathrm{S}} \mathbf{v} \cdot \boldsymbol{\psi}=\int_{\Omega_{\mathrm{S}}} \rho_{\mathrm{S}} \mathbf{g} \cdot \boldsymbol{\psi}+\int_{\Gamma_{\mathrm{I}}} p \boldsymbol{\psi} \cdot \boldsymbol{\nu} d \Gamma, \quad \forall \boldsymbol{\psi} \in H_{\Gamma_{\mathrm{D}}}^{1}\left(\Omega_{\mathrm{S}}\right)^{2}
$$

Hence, $\mathbf{v}$ is the solution (in the sense of distributions) of the following elasticity problem:

$$
\begin{aligned}
-\operatorname{div}[\boldsymbol{\sigma}(\mathbf{v})]+\rho_{\mathrm{S}} \mathbf{v} & =\rho_{\mathrm{S}} \mathbf{g}, & & \text { in } \Omega_{\mathrm{S}} \\
\boldsymbol{\sigma}(\mathbf{v}) \boldsymbol{\nu} & =\left(-p+\rho_{\mathrm{F}} g \mathbf{k} \cdot \boldsymbol{\nu} \mathbf{v} \cdot \boldsymbol{\nu}\right) \boldsymbol{\nu}, & & \text { on } \Gamma_{\mathrm{I}}, \\
\boldsymbol{\sigma}(\mathbf{v}) \mathbf{n} & =\mathbf{0}, & & \text { on } \Gamma_{\mathrm{N}} \\
\mathbf{v} & =\mathbf{0}, & & \text { on } \Gamma_{\mathrm{D}} .
\end{aligned}
$$

Therefore, according to [13], we know that $\mathbf{v} \in H^{1+t}\left(\Omega_{\mathrm{S}}\right)^{2}$, with $t \in(0,1]$ depending on the reentrant corners of $\partial \Omega_{\mathrm{S}}$, on the angles between $\Gamma_{\mathrm{N}}, \Gamma_{\mathrm{D}}$ and $\Gamma_{\mathrm{I}}$, and on the Lamé coefficients $\lambda_{\mathrm{S}}$ and $\mu_{\mathrm{S}}$, and

$$
\|\mathbf{v}\|_{H^{1+t}\left(\Omega_{\mathrm{S}}\right)^{2}} \leq C\left[\rho_{\mathrm{S}}\|\mathbf{g}\|_{L^{2}\left(\Omega_{\mathrm{F}}\right)^{2}}+\|p\|_{H^{1 / 2}\left(\Gamma_{\mathrm{I}}\right)}+\rho_{\mathrm{F}} g \sum_{j=1}^{J}\|\mathbf{v} \cdot \boldsymbol{\nu}\|_{H^{1 / 2}\left(\Gamma_{j}\right)}\right] \leq C|(\mathbf{f}, \mathbf{g})|,
$$

concluding the lemma.

Finally, further regularity can be proved for the eigenfunctions of our problem:

Theorem 4.3. Let $(\mathbf{u}, \mathbf{v})$ be an eigenfunction of $\mathbf{T}$ associated with an eigenvalue $\lambda \in(0,1)$. Let $p \in Q$ be such that $(\mathbf{u}, \mathbf{v}, p)$ is the corresponding eigenfunction of (3.4)-(3.5). Then $\mathbf{u} \in H^{s}\left(\Omega_{\mathrm{F}}\right)^{2}, \mathbf{u} \cdot \boldsymbol{\nu} \in H^{1 / 2+s}\left(\Gamma_{\mathrm{O}}\right)$, $\mathbf{v} \in H^{1+t}\left(\Omega_{\mathrm{S}}\right)^{2}, p \in H^{1+s}\left(\Omega_{\mathrm{F}}\right)$ and

$$
\|\mathbf{u}\|_{H^{s}\left(\Omega_{\mathrm{F}}\right)^{2}}+\|\mathbf{u} \cdot \boldsymbol{\nu}\|_{H^{1 / 2+s}\left(\Gamma_{\mathrm{O}}\right)}+\|p\|_{H^{1+s}\left(\Omega_{\mathrm{F}}\right)}+\|\mathbf{v}\|_{H^{1+t}\left(\Omega_{\mathrm{S}}\right)^{2}} \leq C|(\mathbf{u}, \mathbf{v})|,
$$

with $s$ and $t$ as in Lemmas 4.2 and 4.3, respectively, and $C$ not depending on $c$.

Proof. Since $\lambda \neq 1$, because of Theorem 4.2, $(\mathbf{u}, \mathbf{v}) \in \mathbf{G}$. Now, $(\mathbf{u}, \mathbf{v}, p)$ is the solution of problem $(3.9-$ 3.10) with $(\mathbf{f}, \mathbf{g})=\frac{1}{\lambda}(\mathbf{u}, \mathbf{v}) \in \mathbf{G} \subset \mathbf{K}^{\perp_{\mathrm{H}}}$. Therefore, Lemmas 4.2 and 4.3 apply. Moreover, because of (4.4), $\nabla p+\rho_{\mathrm{F}} \mathbf{u}=\lambda \rho_{\mathrm{F}} \mathbf{u}$; so, $p \in H^{1+s}\left(\Omega_{\mathrm{F}}\right)$ with $\|p\|_{H^{1+s}\left(\Omega_{\mathrm{F}}\right)} \leq C|(\mathbf{u}, \mathbf{v})|$. Finally, as shown in the proof of Lemma 4.2, 
$\mathbf{u} \cdot \boldsymbol{\nu}=\frac{1}{\rho_{\mathrm{F}} g} p$ on $\Gamma_{\mathrm{O}}$; hence $\mathbf{u} \cdot \boldsymbol{\nu} \in H^{1 / 2+s}\left(\Gamma_{\mathrm{O}}\right)$ and $\|\mathbf{u} \cdot \boldsymbol{\nu}\|_{H^{1 / 2+s}\left(\Gamma_{\mathrm{O}}\right)} \leq C|(\mathbf{u}, \mathbf{v})|$, allowing to conclude the theorem.

\section{Finite Element Discretization}

In the previous section it was shown that $\left.\mathbf{T}\right|_{\mathbf{G}}$ is compact, but a standard discretization of $\mathbf{G}$ would require to use finite element spaces of irrotational functions. To avoid it, we will deal with the non compact operator $\left.\mathbf{T}\right|_{\mathbf{V}}$ instead. This operator has an infinite dimensional eigenspace $\mathbf{K}$ consisting of pure rotational motions with no physical entity. However, any suitable numerical approximation should take care of them; otherwise, spurious modes would appear.

In [3] a discretization which does not present spurious modes is introduced for the case of a compressible fluid, but neglecting the gravity effects. An extension to incompressible fluids has been made in [4], where error estimates independent of the acoustic speed have been obtained. The same discretization can be used in our case.

Let $\left\{\mathcal{T}_{h}\right\}$ be a family of regular triangulations of $\Omega_{\mathrm{F}} \cup \Omega_{\mathrm{S}}$ such that every triangle is completely contained either in $\Omega_{\mathrm{F}}$ or in $\Omega_{\mathrm{S}}$ and such that the end points of $\Gamma_{\mathrm{D}}, \Gamma_{\mathrm{N}}, \Gamma_{\mathrm{I}}$ and $\Gamma_{\mathrm{O}}$ coincide with nodes of the triangulation.

For each component of the displacements in the solid we use the standard piecewise linear finite element space

$$
L_{h}\left(\Omega_{\mathrm{S}}\right):=\left\{v \in H^{1}\left(\Omega_{\mathrm{S}}\right):\left.v\right|_{T} \in \mathcal{P}_{1}(T), \forall T \in \mathcal{T}_{h}, T \subset \Omega_{\mathrm{S}}\right\}
$$

and, for the fluid, the Raviart-Thomas space [20]

$$
\mathbf{R}_{h}\left(\Omega_{\mathrm{F}}\right):=\left\{\mathbf{u} \in H\left(\operatorname{div}, \Omega_{\mathrm{F}}\right):\left.\mathbf{u}\right|_{T} \in \mathcal{R}_{0}(T), \forall T \in \mathcal{T}_{h}, T \subset \Omega_{\mathrm{F}}\right\}
$$

where

$$
\mathcal{R}_{0}(T):=\left\{\mathbf{u} \in \mathcal{P}_{1}(T)^{2}: \mathbf{u}(x, y)=(a+b x, c+b y), a, b, c \in \mathbb{R}\right\} .
$$

The discrete analogue of $\mathbf{X}$ is

$$
\mathbf{X}_{h}:=\left\{(\mathbf{u}, \mathbf{v}) \in \mathbf{R}_{h}\left(\Omega_{\mathrm{F}}\right) \times L_{h}\left(\Omega_{\mathrm{S}}\right)^{2}:\left.\mathbf{v}\right|_{\Gamma_{\mathrm{D}}}=\mathbf{0}\right\}
$$

The conforming finite element spaces $\mathbf{V} \cap \mathbf{X}_{h}$ are not adequate for our problem (see [4]). Instead, we use the following ones:

$$
\mathbf{V}_{h}:=\left\{(\mathbf{u}, \mathbf{v}) \in \mathbf{X}_{h}: \int_{\ell}(\mathbf{u} \cdot \boldsymbol{\nu}-\mathbf{v} \cdot \boldsymbol{\nu}) d \Gamma=0, \forall \ell \subset \Gamma_{\mathrm{I}}, \ell \text { edge of } T, T \in \mathcal{T}_{h}\right\} .
$$

Let us remark that, since $\mathbf{V}_{h} \not \subset \mathbf{V}$, our method is non conforming. Finally, for the pressures we use the space of piecewise constant functions

$$
Q_{h}:=\left\{p \in L^{2}\left(\Omega_{\mathrm{F}}\right):\left.p\right|_{T} \in \mathcal{P}_{0}(T), \forall T \in \mathcal{T}_{h}, T \subset \Omega_{\mathrm{F}}\right\} .
$$

Let $\mathbf{W}_{h}:=\left\{(\mathbf{u}, \mathbf{v}) \in \mathbf{V}_{h}: b((\mathbf{u}, \mathbf{v}), q)=0, \forall q \in Q_{h}\right\}=\left\{(\mathbf{u}, \mathbf{v}) \in \mathbf{V}_{h}: \operatorname{div} \mathbf{u}=0\right\}$. The lemma below shows that the bilinear forms $a$ and $b$ satisfy both Brezzi's conditions on these finite element spaces.

Lemma 5.1. The bilinear forms $a$ and $b$ satisfy:

i) a is coercive on $\mathbf{W}_{h}$;

ii) there exists $\beta>0$, not depending on $h$, such that

$$
\inf _{\substack{q \in Q_{h} \\ q \neq 0}} \sup _{\substack{(\mathbf{u}, \mathbf{v}) \in \mathbf{V}_{h} \\(\mathbf{u}, \mathbf{v}) \neq(\mathbf{0}, \mathbf{0})}} \frac{b((\mathbf{u}, \mathbf{v}), q)}{\|(\mathbf{u}, \mathbf{v})\||q|} \geq \beta .
$$


Proof. Since $\mathbf{W}_{h} \not \subset \mathbf{V}$, the coerciveness of $a$ on this space does not follow from Lemma 3.1. However, it is enough to show that for all $(\mathbf{u}, \mathbf{v}) \in \mathbf{V}_{h},\|\mathbf{u} \cdot \boldsymbol{\nu}\|_{L^{2}\left(\Gamma_{\mathrm{I}}\right)}^{2} \leq\|\mathbf{v} \cdot \boldsymbol{\nu}\|_{L^{2}\left(\Gamma_{\mathrm{I}}\right.}^{2}$. For this, since $\mathbf{u} \cdot \boldsymbol{\nu}$ is constant on each edge $\ell \subset \Gamma_{\mathrm{I}}$, from the definition of $\mathbf{V}_{h}$ we have

$$
\|\mathbf{u} \cdot \boldsymbol{\nu}\|_{L^{2}\left(\Gamma_{\mathrm{I}}\right)}^{2}=\sum_{\ell \subset \Gamma_{\mathrm{I}}}|\ell|\left(\frac{1}{|\ell|} \int_{\ell} \mathbf{v} \cdot \boldsymbol{\nu} d \Gamma\right)^{2} \leq\|\mathbf{v} \cdot \boldsymbol{\nu}\|_{L^{2}\left(\Gamma_{\mathrm{I}}\right)}^{2} \leq C\|\mathbf{v}\|_{H_{\Gamma_{\mathrm{D}}}^{1}\left(\Omega_{\mathrm{S}}\right)^{2}}^{2},
$$

where $|\ell|$ denotes the length of that edge.

To prove the inf-sup condition we are going to show that for all $q \in Q_{h}$ there exists $\left(\mathbf{u}_{h}, \mathbf{v}_{h}\right) \in \mathbf{V}_{h}$ satisfying

$$
\operatorname{div} \mathbf{u}_{h}=q \quad \text { in } \Omega_{\mathrm{F}} \quad \text { and } \quad\left\|\left(\mathbf{u}_{h}, \mathbf{v}_{h}\right)\right\| \leq C|q| .
$$

Let $(\mathbf{u}, \mathbf{v}) \in \mathbf{V}$ be defined as in the proof of Lemma 3.1. Since $Q_{h} \subset Q$, then (u, v) satisfies (3.7). Let $\mathbf{v}_{h} \in L_{h}\left(\Omega_{\mathrm{S}}\right)^{2}$ be a Clement's interpolant of $\mathbf{v}$, vanishing on $\Gamma_{\mathrm{D}} \cup \Gamma_{\mathrm{N}}$ (see [9]); then

$$
\left\|\mathbf{v}_{h}\right\|_{H^{1}\left(\Omega_{\mathrm{S}}\right)^{2}} \leq C\|\mathbf{v}\|_{H^{1}\left(\Omega_{\mathrm{S}}\right)^{2}} \leq C|q|,
$$

the last inequality because of (3.7).

Let $c_{0} \in \mathbb{R}$ be such that the following Neumann problem is compatible:

$$
\begin{aligned}
& \Delta \varphi=q, \quad \text { in } \Omega_{\mathrm{F}}, \\
& \frac{\partial \varphi}{\partial \boldsymbol{\nu}}=\mathbf{v}_{h} \cdot \boldsymbol{\nu}, \quad \text { on } \Gamma_{\mathrm{I}}, \\
& \frac{\partial \varphi}{\partial \boldsymbol{\nu}}=c_{0}, \quad \text { on } \Gamma_{\mathrm{O}} .
\end{aligned}
$$

Hence,

and then, because of $(5.2)$,

$$
c_{0}=\frac{1}{\left|\Gamma_{\mathrm{O}}\right|}\left(\int_{\Omega_{\mathrm{F}}} q-\int_{\Gamma_{\mathrm{I}}} \mathbf{v}_{h} \cdot \boldsymbol{\nu} d \Gamma\right)
$$

$$
\left|c_{0}\right| \leq C|q| \text {. }
$$

Let $\varphi$ be a solution of problem (5.3-5.5). Because of the usual a priori estimate, $\varphi \in H^{1+s}\left(\Omega_{\mathrm{F}}\right)$, with $s \geq 1 / 2$ as in Lemma 4.2 , and

$$
\|\nabla \varphi\|_{H^{s}\left(\Omega_{\mathrm{F}}\right)^{2}} \leq C\left[|q|+\sum_{j=1}^{J}\left\|\mathbf{v}_{h} \cdot \boldsymbol{\nu}\right\|_{H^{1 / 2}\left(\Gamma_{j}\right)}+\left\|c_{0}\right\|_{H^{1 / 2}\left(\Gamma_{\mathrm{O}}\right)}\right] \leq C|q|,
$$

the last inequality being true because of (5.2), (5.6) and the fact that $c_{0}$ is constant.

Let $\mathbf{u}_{h} \in \mathbf{R}_{h}\left(\Omega_{\mathrm{F}}\right)$ be the Raviart-Thomas interpolant of $\nabla \varphi$ (see [8]). Then, for each edge $\ell \subset \Gamma_{\mathrm{I}}$,

$$
\int_{\ell} \mathbf{u}_{h} \cdot \boldsymbol{\nu} d \Gamma=\int_{\ell} \frac{\partial \varphi}{\partial \boldsymbol{\nu}} d \Gamma=\int_{\ell} \mathbf{v}_{h} \cdot \boldsymbol{\nu} d \Gamma
$$

hence, $\left(\mathbf{u}_{h}, \mathbf{v}_{h}\right) \in \mathbf{V}_{h}$.

On the other hand, for those edges $\ell \subset \Gamma_{\mathrm{O}}$ we have

$$
\left.\left(\mathbf{u}_{h} \cdot \boldsymbol{\nu}\right)\right|_{\ell}=\frac{1}{|\ell|} \int_{\ell} \frac{\partial \varphi}{\partial \boldsymbol{\nu}} d \Gamma=c_{0}
$$


and then

$$
\left\|\mathbf{u}_{h} \cdot \boldsymbol{\nu}\right\|_{L^{2}\left(\Gamma_{\mathrm{F}}\right)}^{2}=\sum_{\ell \subset \Gamma_{\mathrm{F}}}|\ell|\left(\frac{1}{|\ell|} \int_{\ell} \frac{\partial \varphi}{\partial \boldsymbol{\nu}} d \Gamma\right)^{2} \leq\left\|\mathbf{v}_{h} \cdot \boldsymbol{\nu}\right\|_{L^{2}\left(\Gamma_{\mathrm{I}}\right)}^{2}+\left|c_{0}\right|^{2}\left|\Gamma_{\mathrm{O}}\right| \leq C|q|^{2}
$$

Now, by the stability property of this interpolation,

$$
\left\|\mathbf{u}_{h}\right\|_{H\left(\operatorname{div}, \Omega_{\mathrm{F}}\right)} \leq C\left[\|\nabla \varphi\|_{H^{s}\left(\Omega_{\mathrm{F}}\right)^{2}}+\|\operatorname{div}(\nabla \varphi)\|_{L^{2}\left(\Omega_{\mathrm{F}}\right)}\right] \leq C|q|,
$$

and by the classical property of Raviart-Thomas interpolation, $\operatorname{div} \mathbf{u}_{h}$ is the $L^{2}\left(\Omega_{\mathrm{F}}\right)$ projection of $\operatorname{div}(\nabla \varphi)=q$ onto $Q_{h}$; hence, since $q \in Q_{h}$, then $\operatorname{div} \mathbf{u}_{h}=q$.

Finally, (5.2), (5.10) and (5.11) show that $\left(\mathbf{u}_{h}, \mathbf{v}_{h}\right)$ satisfies the inequality in (5.1), which allows us to conclude the lemma.

Remark 5.1. The previous proof is valid provided $\Gamma_{\mathrm{o}} \neq \emptyset$. However, for similar problems without a free boundary of the fluid $\Gamma_{\mathrm{O}}$, the result is also true. In fact, the proof of Theorem 5.1 in [4] can be repeated in this case and this, plus an additional inequality similar to (5.10) (needed to take care of the gravity effects), prove the result.

Remark 5.2. If the fluid domains has an interior angle of $2 \pi$, the constant $s$ in (5.7) takes the limit value $s=1 / 2$ and then $\frac{\partial \varphi}{\partial \nu}$ is not well defined as an $L^{2}\left(\Gamma_{\mathrm{F}}\right)$ function. However, the integral $\int_{\ell} \frac{\partial \varphi}{\partial \nu} d \Gamma$ makes sense as stated in Section III.3.3 of [8]. In fact, since $H^{s}\left(\Omega_{\mathrm{F}}\right) \hookrightarrow L^{2 /(1-s)}\left(\Omega_{\mathrm{F}}\right)$ and $\operatorname{div}(\nabla \varphi) \in L^{2}\left(\Omega_{\mathrm{F}}\right)$, it is easy to check that these normal derivatives are well defined in the dual space $\left[W^{1-1 / r, r}\left(\Gamma_{\mathrm{F}}\right)\right]^{\prime}$ for $r=\frac{2}{1+s} \in(1,2)$. In this case, the integral $\int_{\ell} \frac{\partial \varphi}{\partial \boldsymbol{\nu}} d \Gamma$ in equations (5.8) and (5.9) must be understood in the sense of the duality pairing

$$
\left\langle\frac{\partial \varphi}{\partial \boldsymbol{\nu}}, \chi_{\ell}\right\rangle_{\left[W^{1-1 / r, r}\left(\Gamma_{\mathrm{F}}\right)\right]^{\prime} \times W^{1-1 / r, r}\left(\Gamma_{\mathrm{F}}\right)}
$$

with $\chi_{\ell}$ being the characteristic function of the edge $\ell$ which is known to satisfy $\chi_{\ell} \in W^{1-1 / r, r}\left(\Gamma_{\mathrm{F}}\right)$, for any $r \in(1,2)$.

As a consequence of Lemma 5.1, by applying the standard theory of mixed methods (see, for instance, [8]) we know that for any $(\mathbf{f}, \mathbf{g}) \in \mathbf{H}$, there exists a unique solution $\left(\mathbf{u}_{h}, \mathbf{v}_{h}, p_{h}\right) \in \mathbf{V}_{h} \times Q_{h}$ of the discrete source problem:

$$
\begin{array}{clrl}
a\left(\left(\mathbf{u}_{h}, \mathbf{v}_{h}\right),(\boldsymbol{\phi}, \boldsymbol{\psi})\right)+b\left((\boldsymbol{\phi}, \boldsymbol{\psi}), p_{h}\right) & =d((\mathbf{f}, \mathbf{g}),(\boldsymbol{\phi}, \boldsymbol{\psi})), & & \forall(\boldsymbol{\phi}, \boldsymbol{\psi}) \in \mathbf{V}_{h}, \\
b\left(\left(\mathbf{u}_{h}, \mathbf{v}_{h}\right), q\right)-\frac{1}{\rho_{\mathrm{F}} c^{2}} \int_{\Omega_{\mathrm{F}}} p_{h} q=0, & & \forall q \in Q_{h},
\end{array}
$$

and, moreover,

$$
\left\|\left(\mathbf{u}_{h}, \mathbf{v}_{h}\right)\right\|+\left|p_{h}\right| \leq C|(\mathbf{f}, \mathbf{g})|
$$

with a constant $C$ independent of $h$ and $c$ (even for $c=\infty$ ).

Now, we can define a discrete analogue of $\mathbf{T}$ : let $\mathbf{T}_{h}: \mathbf{H} \longrightarrow \mathbf{V}_{h}$ be defined by $\mathbf{T}_{h}(\mathbf{f}, \mathbf{g})=\left(\mathbf{u}_{h}, \mathbf{v}_{h}\right)$, with $\left(\mathbf{u}_{h}, \mathbf{v}_{h}, p_{h}\right)$ being the solution of (5.12)-(5.13); because of (5.14), the operators $\mathbf{T}_{h}$ are bounded uniformly on $h$ and $c$. In the following section we show that the spectra of these operators provide optimal order approximations of the spectrum of $\mathbf{T}$. 


\section{Spectral approximation}

In [4] and [18] the spectral approximation theory for noncompact operators from [11] has been adapted to the nonconforming case to obtain error estimates for other simpler fluid-structure vibration problems. This theory can be extended to cover our problem, however it does not seem to provide optimal error estimates.

In fact, the theory in [18] yields error estimates for the computed eigenfunctions and eigenvalues in terms of $\left\|\left(\mathbf{T}-\mathbf{T}_{h}\right) \mid \mathbf{v}_{h}\right\|$. In our case, it can be proved that $\left\|\left(\mathbf{T}-\mathbf{T}_{h}\right) \mid \mathbf{V}_{h}\right\| \leq C h^{r}$, but with $r:=\min \left\{\frac{1}{2}, t\right\}$ instead of $r:=\min \{s, t\}$ as in [4] ( $s$ and $t$ being the constants of Lemmas 4.2 and 4.3, respectively). Thus, such procedure yields non optimal error estimates when $t$ and $s$ are both greater than $1 / 2$.

On the other hand, the standard spectral theory for compact operators as stated in [1] (Section 7) cannot be directly applied to our problem. In fact, $\mathbf{T}$ is not compact since it is the identity when restricted to the infinite dimensional subspace $\mathbf{K}$. However, as we show below, $\lim _{h \rightarrow 0}\left\|\left.\left(\mathbf{T}-\mathbf{T}_{h}\right)\right|_{\mathbf{K}^{\perp} \mathrm{H}}\right\|=0$, and this can be exploited to show that the spectrum and the eigenfunctions of $\mathbf{T}_{h}$ approximate those of $\mathbf{T}$ with optimal order.

Firstly, let us show that the discrete operators $\mathbf{T}_{h}$ have eigenspaces providing good approximations of the infinite dimensional eigenspace $\mathbf{K}$ of $\mathbf{T}$, and with exactly the same eigenvalue.

Theorem 6.1. $\lambda=1$ is an eigenvalue of $\mathbf{T}_{h}$ with corresponding eigenspace $\mathbf{K}_{h}=\mathbf{K} \cap \mathbf{V}_{h}$.

Proof. The proof is omitted since it is essentially the same as that of Theorem 4.2 in [3].

Notice that the discrete eigenspace $\mathbf{K}_{h}$ can also be written

$$
\mathbf{K}_{h}=\left\{(\operatorname{curl} \xi, \mathbf{0}): \xi \in L_{h}\left(\Omega_{\mathrm{F}}\right) \text { and } \xi \text { is constant on each connected component of } \Gamma_{\mathrm{F}}\right\} ;
$$

therefore, for any eigenfunction $(\operatorname{curl} \xi, \mathbf{0}) \in \mathbf{K}$ there exist discrete eigenfunctions $\left(\operatorname{curl} \xi_{h}, \mathbf{0}\right) \in \mathbf{K}_{h}$ such that $\left\|\left(\operatorname{curl} \xi_{h}, \mathbf{0}\right)-(\operatorname{curl} \xi, \mathbf{0})\right\| \rightarrow 0$ as $h \rightarrow 0$. In fact, this is for instance true if $\xi_{h} \in L_{h}\left(\Omega_{\mathrm{F}}\right)$ is chosen as a Clement's interpolant of $\xi$ preserving its constant boundary values on each connected component of $\Gamma_{\mathrm{F}}$ (see [9]).

Secondly, let us show that smooth functions of $\mathbf{V}$ can be well approximated from $\mathbf{V}_{h}$. In [3] a $\mathbf{V}_{h}$-interpolant operator $\mathbf{I}_{h}$ is introduced; it consists of the Lagrange interpolant of the solid displacements and the RaviartThomas interpolant of the fluid displacements, the latter conveniently modified on the interface edges in order to allow for $\mathbf{I}_{h}(\mathbf{u}, \mathbf{v}) \in \mathbf{V}_{h}$.

The same interpolation is useful in our case. In fact, for any $r \in(0,1)$ let $\mathbf{V}_{r}$ denote the subspace of $\mathbf{V}$ defined by

$$
\mathbf{V}_{r}:=\left\{(\mathbf{u}, \mathbf{v}) \in \mathbf{V}: \mathbf{u} \in H^{r}\left(\Omega_{\mathrm{F}}\right)^{2}, \operatorname{div} \mathbf{u} \in H^{r}\left(\Omega_{\mathrm{F}}\right), \mathbf{u} \cdot \boldsymbol{\nu} \in H^{r}\left(\Gamma_{\mathrm{O}}\right) \text { and } \mathbf{v} \in H^{1+r}\left(\Omega_{\mathrm{S}}\right)^{2}\right\}
$$

and $\|\cdot\|_{r}$ the natural norm of this space; we have the following approximation result.

Lemma 6.1. There exists a linear operator $\mathbf{I}_{h}: \mathbf{V}_{r} \longrightarrow \mathbf{V}_{h}$ and a strictly positive constant $C$ such that for all $(\mathbf{u}, \mathbf{v}) \in \mathbf{V}_{r}$,

$$
\left\|(\mathbf{u}, \mathbf{v})-\mathbf{I}_{h}(\mathbf{u}, \mathbf{v})\right\| \leq C h^{r}\|(\mathbf{u}, \mathbf{v})\|_{r}
$$

Proof. The arguments in Theorem 5.2 and Lemma 5.1 of [3] can be easily modified to prove that the interpolant operator defined therein satisfy $(6.1)$ with the norm $\|\cdot\|$ including the $L^{2}\left(\Gamma_{\mathrm{F}}\right)$ terms and for any $r>0$.

Now we are able to prove that $\left\|\left.\left(\mathbf{T}-\mathbf{T}_{h}\right)\right|_{\mathbf{K}^{\perp_{\mathrm{H}}}}\right\|$ converges to zero as $h$ goes to zero, and with optimal order when restricted to an eigenspace of $\mathbf{T}$.

Lemma 6.2. There exists a strictly positive constant $C$, depending neither on $h$ nor on $c$, such that for all $(\mathbf{f}, \mathbf{g}) \in \mathbf{K}^{\perp_{\mathrm{H}}}$

$$
\left\|\left(\mathbf{T}-\mathbf{T}_{h}\right)(\mathbf{f}, \mathbf{g})\right\| \leq C h^{r}|(\mathbf{f}, \mathbf{g})|,
$$


with $r:=\min \left\{\frac{1}{2}, t\right\}$. Furthermore, if $(\mathbf{f}, \mathbf{g}) \in \mathbf{K}^{\perp_{\mathrm{H}}}$ is an eigenfunction of $\mathbf{T}$ then $r:=\min \{s, t\}$. The constants $s \in\left[\frac{1}{2}, 1\right]$ and $t \in(0,1]$ are those of Lemmas 4.2 and 4.3, respectively.

Proof. Let $(\mathbf{u}, \mathbf{v}):=\mathbf{T}(\mathbf{f}, \mathbf{g})$ and $p \in Q$ be such that $(\mathbf{u}, \mathbf{v}, p)$ is the solution of the mixed problem (3.9)-(3.10). Analogously, let $\left(\mathbf{u}_{h}, \mathbf{v}_{h}\right):=\mathbf{T}_{h}(\mathbf{f}, \mathbf{g})$ and $p_{h} \in Q_{h}$ such that $\left(\mathbf{u}_{h}, \mathbf{v}_{h}, p_{h}\right)$ is the solution of the discrete problem (5.12)-(5.13). Since $\mathbf{V}_{h}$ is not a subspace of $\mathbf{V},\left(\mathbf{u}_{h}, \mathbf{v}_{h}, p_{h}\right)$ is a nonconforming finite element approximate solution of (3.9)-(3.10); however, the standard theory can be easily adapted to cover this case.

Following the lines of [8] (Sections II.2.4 and II.2.6), by using that $\mathbf{V}_{h}$ and $Q_{h}$ satisfy both Brezzi's conditions (Lemma 5.1), it is easy to show that

$$
\begin{aligned}
\left\|(\mathbf{u}, \mathbf{v})-\left(\mathbf{u}_{h}, \mathbf{v}_{h}\right)\right\|+\left|p-p_{h}\right| \leq & C\left[\operatorname{dist}\left((\mathbf{u}, \mathbf{v}), \mathbf{V}_{h}\right)+\operatorname{dist}\left(p, Q_{h}\right)\right. \\
& \left.+\sup _{\substack{\left.(\phi, \psi) \in \mathbf{V}_{h} \\
\phi, \psi\right) \neq(\mathbf{0}, \mathbf{0})}} \frac{a((\mathbf{u}, \mathbf{v}),(\boldsymbol{\phi}, \boldsymbol{\psi}))+b(p,(\boldsymbol{\phi}, \boldsymbol{\psi}))-d((\mathbf{f}, \mathbf{g}),(\boldsymbol{\phi}, \boldsymbol{\psi}))}{\|(\boldsymbol{\phi}, \boldsymbol{\psi})\|}\right],
\end{aligned}
$$

with a constant $C$ depending neither on $c$ nor on $h$.

Now, for any $(\mathbf{f}, \mathbf{g}) \in \mathbf{K}^{\perp_{\mathrm{H}}}$, by virtue of Lemmas 4.2 and 4.3 we have that

$$
\|\mathbf{u}\|_{H^{s}\left(\Omega_{\mathrm{F}}\right)^{2}}+\|\mathbf{u} \cdot \boldsymbol{\nu}\|_{H^{1 / 2}\left(\Gamma_{\mathrm{O}}\right)}+\|p\|_{H^{1}\left(\Omega_{\mathrm{F}}\right)}+\|\mathbf{v}\|_{H^{1+t}\left(\Omega_{\mathrm{S}}\right)^{2}} \leq C|(\mathbf{f}, \mathbf{g})|
$$

(with $C$ independent of $c$ ), whereas if $(\mathbf{f}, \mathbf{g})$ is an eigenfunction of $\mathbf{T}$, Theorem 4.3 provides further regularity for $\mathbf{u} \cdot \boldsymbol{\nu}$ and $p$ :

$$
\|\mathbf{u}\|_{H^{s}\left(\Omega_{\mathrm{F}}\right)^{2}}+\|\mathbf{u} \cdot \boldsymbol{\nu}\|_{H^{1 / 2+s}\left(\Gamma_{\mathrm{O}}\right)}+\|p\|_{H^{1+s}\left(\Omega_{\mathrm{F}}\right)}+\|\mathbf{v}\|_{H^{1+t}\left(\Omega_{\mathrm{S}}\right)^{2}} \leq C|(\mathbf{f}, \mathbf{g})| .
$$

Therefore, we have

$$
\operatorname{dist}\left(p, Q_{h}\right) \leq C h\|p\|_{H^{1}\left(\Omega_{\mathrm{F}}\right)} \leq C h|(\mathbf{f}, \mathbf{g})|
$$

in both cases and, since $\operatorname{div} \mathbf{u}=\frac{1}{\rho_{\mathrm{F}} c^{2}} p$ (because of (3.10)), by applying Lemma 6.1,

$$
\operatorname{dist}\left((\mathbf{u}, \mathbf{v}), \mathbf{V}_{h}\right) \leq C h^{r}|(\mathbf{f}, \mathbf{g})|
$$

with $r=\min \left\{\frac{1}{2}, t\right\}$ for any $(\mathbf{f}, \mathbf{g}) \in \mathbf{K}^{\perp_{\mathrm{H}}}$, and $r=\min \{s, t\}$ if $(\mathbf{f}, \mathbf{g}) \in \mathbf{K}^{\perp_{\mathrm{H}}}$ is an eigenfunction of $\mathbf{T}$.

The remaining consistency term in the right hand side of (6.2) can be bounded proceeding as in Lemma 5.7 of [3]; by so doing, we obtain for all $(\boldsymbol{\phi}, \boldsymbol{\psi}) \in \mathbf{V}_{h}$

$$
\begin{aligned}
|a((\mathbf{u}, \mathbf{v}),(\boldsymbol{\phi}, \boldsymbol{\psi}))+b(p,(\boldsymbol{\phi}, \boldsymbol{\psi}))-d((\mathbf{f}, \mathbf{g}),(\boldsymbol{\phi}, \boldsymbol{\psi}))| & =\left|\int_{\Gamma_{\mathrm{I}}} p(\boldsymbol{\phi} \cdot \boldsymbol{\nu}-\boldsymbol{\psi} \cdot \boldsymbol{\nu}) d \Gamma\right| \\
& \leq C h\|p\|_{H^{1}\left(\Omega_{\mathrm{F}}\right)}\|(\boldsymbol{\phi}, \boldsymbol{\psi})\| \leq C h|(\mathbf{f}, \mathbf{g})|\|(\boldsymbol{\phi}, \boldsymbol{\psi})\| .
\end{aligned}
$$

Therefore, by replacing (6.3), (6.4) and (6.5) in (6.2), we obtain $\left\|\left(\mathbf{T}-\mathbf{T}_{h}\right)(\mathbf{f}, \mathbf{g})\right\|=\left\|(\mathbf{u}, \mathbf{v})-\left(\mathbf{u}_{h}, \mathbf{v}_{h}\right)\right\| \leq$ $C h^{r}|(\mathbf{f}, \mathbf{g})|$, and conclude the lemma.

Remark 6.1. As a byproduct of the proof of this Lemma (see (6.2)), the following error estimate for the approximation of the pressure has been also proved:

$$
\left|p-p_{h}\right| \leq C h^{r}|(\mathbf{f}, \mathbf{g})|
$$

with $r \in(0,1]$ as in Lemma 6.2 . 
Notice that since $\lambda=1$ is an eigenvalue of $\mathbf{T}$ with corresponding eigenspace $\mathbf{K}$ and $\mathbf{G}=\mathbf{K}^{\perp} \mathrm{V}$ is an invariant subspace of $\mathbf{T}$, then $\sigma\left(\left.\mathbf{T}\right|_{\mathbf{V}}\right)=\{1\} \cup \sigma\left(\left.\mathbf{T}\right|_{\mathbf{G}}\right)$.

Analogously, $\lambda=1$ is an eigenvalue of $\mathbf{T}_{h}$ with corresponding eigenspace $\mathbf{K}_{h}=\mathbf{K} \cap \mathbf{V}_{h}$. Let $\mathbf{G}_{h}$ denote the orthogonal complement of $\mathbf{K}_{h}$ in $\mathbf{V}_{h}$. It is easy to check that $\mathbf{G}_{h}:=\mathbf{K}_{h}^{\perp_{\mathrm{h}}}=\mathbf{K}^{\perp_{\mathrm{H}}} \cap \mathbf{V}_{h}$ and that $\mathbf{G}_{h}$ and $\mathbf{K}_{h}$ are also orthogonal with respect to the bilinear form $d$. Hence, since $\mathbf{T}_{h}$ is self-adjoint with respect to $d$, then $\mathbf{G}_{h}$ is an invariant subspace of $\mathbf{T}_{h}$. Thus we also have $\sigma\left(\left.\mathbf{T}_{h}\right|_{\mathbf{V}_{h}}\right)=\{1\} \cup \sigma\left(\left.\mathbf{T}_{h}\right|_{\mathbf{G}_{h}}\right)$.

We want to show that the spectrum and the eigenfunctions of $\left.\mathbf{T}_{h}\right|_{\mathbf{G}_{h}}$ approximate those of $\left.\mathbf{T}\right|_{\mathbf{G}}$. For this, it is convenient to define the operators $\widetilde{\mathbf{T}}:=\mathbf{T} \circ \mathbf{P}$ and $\widetilde{\mathbf{T}}_{h}:=\mathbf{T}_{h} \circ \mathbf{P}_{h}$, where $\mathbf{P}: \mathbf{H} \longmapsto \mathbf{K}^{\perp_{\mathrm{H}}}$ and $\mathbf{P}_{h}: \mathbf{H} \longmapsto \mathbf{K}_{h}^{\perp_{\mathrm{H}}}$ are the $\mathbf{H}$-orthogonal projections onto $\mathbf{K}^{\perp_{\mathrm{H}}}$ and $\mathbf{K}_{h}^{\perp_{\mathrm{H}}}$, respectively. Then $\left.\widetilde{\mathbf{T}}\right|_{\mathbf{K}}=0$ and, since $\mathbf{G} \subset \mathbf{K}^{\perp_{\mathrm{H}}}$, $\left.\widetilde{\mathbf{T}}\right|_{\mathbf{G}}=\left.\mathbf{T}\right|_{\mathbf{G}}$. Analogously, $\left.\widetilde{\mathbf{T}}_{h}\right|_{\mathbf{K}_{h}}=0$ and, since $\mathbf{G}_{h} \subset \mathbf{K}_{h}^{\perp_{\mathrm{H}}},\left.\widetilde{\mathbf{T}}_{h}\right|_{\mathbf{G}_{h}}=\left.\mathbf{T}_{h}\right|_{\mathbf{G}_{h}}$. Therefore, apart from $\lambda=0$ or 1 , the corresponding spectra and eigenfunctions coincide for $\mathbf{T}$ and $\widetilde{\mathbf{T}}$, as well as for $\mathbf{T}_{h}$ and $\widetilde{\mathbf{T}}_{h}$. Furthermore, we have the following approximation result.

Lemma 6.3. There exists a strictly positive constant $C$, depending neither on $h$ nor on $c$, such that for any eigenfunction $(\mathbf{f}, \mathbf{g})$ of $\widetilde{\mathbf{T}}$ corresponding to an eigenvalue $\lambda>0$

$$
\left\|\left(\widetilde{\mathbf{T}}-\widetilde{\mathbf{T}}_{h}\right)(\mathbf{f}, \mathbf{g})\right\| \leq C h^{r}|(\mathbf{f}, \mathbf{g})|,
$$

where $r:=\min \{s, t\}$, with $s \in\left[\frac{1}{2}, 1\right]$ and $t \in(0,1]$ as in Lemmas 4.2 and 4.3, respectively.

Proof. By the definitions of $\widetilde{\mathbf{T}}$ and $\widetilde{\mathbf{T}}_{h}$ it holds that $\left.\widetilde{\mathbf{T}}\right|_{\mathbf{K}^{\perp_{\mathrm{H}}}}=\left.\mathbf{T}\right|_{\mathbf{K}^{\perp_{\mathrm{H}}}}$ and $\left.\widetilde{\mathbf{T}}_{h}\right|_{\mathbf{K}_{h}^{\perp_{\mathrm{H}}}}=\left.\mathbf{T}_{h}\right|_{\mathbf{K}_{h}^{\perp_{\mathrm{H}}}}$. Hence, this lemma is a direct consequence of Lemma 6.2 and the fact that if $\lambda>0$, then $(\mathbf{f}, \mathbf{g}) \in \mathbf{K}^{\perp_{\mathrm{H}}} \subset \mathbf{K}_{h}^{\perp_{\mathrm{H}}}$.

Finally, in order to use the spectral approximation theory in [1], we prove the convergence of $\widetilde{\mathbf{T}}_{h}$ to $\widetilde{\mathbf{T}}$ in norm.

Lemma 6.4. It holds that

$$
\sup _{\substack{(\mathbf{f}, \mathbf{g}) \in \mathbf{H} \\(\mathbf{f}, \mathbf{g}) \neq 0}} \frac{\left\|\left(\widetilde{\mathbf{T}}-\widetilde{\mathbf{T}}_{h}\right)(\mathbf{f}, \mathbf{g})\right\|}{|(\mathbf{f}, \mathbf{g})|} \rightarrow 0, \quad \text { as } h \rightarrow 0 .
$$

Proof. Let $(\mathbf{f}, \mathbf{g}) \in \mathbf{H}$ and consider its orthogonal decomposition

$$
(\mathbf{f}, \mathbf{g})=(\nabla \phi, \mathbf{g})+(\operatorname{curl} \xi, \mathbf{0})
$$

with $(\nabla \phi, \mathbf{g}) \in \mathbf{K}^{\perp_{\mathrm{H}}}$ and $(\operatorname{curl} \xi, \mathbf{0}) \in \mathbf{K}$. Then

$$
\begin{aligned}
\left\|\left(\widetilde{\mathbf{T}}-\widetilde{\mathbf{T}}_{h}\right)(\mathbf{f}, \mathbf{g})\right\| & \leq\left\|\left(\widetilde{\mathbf{T}}-\widetilde{\mathbf{T}}_{h}\right)(\nabla \varphi, \mathbf{g})\right\|+\left\|\left(\widetilde{\mathbf{T}}-\widetilde{\mathbf{T}}_{h}\right)(\operatorname{curl} \xi, \mathbf{0})\right\| \\
& =\left\|\left(\mathbf{T}-\mathbf{T}_{h}\right)(\nabla \varphi, \mathbf{g})\right\|+\left\|\widetilde{\mathbf{T}}_{h}(\operatorname{curl} \xi, \mathbf{0})\right\| .
\end{aligned}
$$

The first term in the right hand side of (6.7) can be bounded by using Lemma 6.2:

$$
\left\|\left(\mathbf{T}-\mathbf{T}_{h}\right)(\nabla \varphi, \mathbf{g})\right\| \leq C h^{r}|(\nabla \varphi, \mathbf{g})| \leq C h^{r}|(\mathbf{f}, \mathbf{g})|, \quad \text { with } r>0 .
$$

To deal with the second term we denote

$$
\left(\mathbf{u}_{h}, \mathbf{v}_{h}\right):=\widetilde{\mathbf{T}}_{h}(\operatorname{curl} \xi, \mathbf{0})=\mathbf{T}_{h}\left(\mathbf{P}_{h}(\operatorname{curl} \xi, \mathbf{0})\right) .
$$

Hence, there exists $p_{h} \in Q_{h}$ such that $\left(\mathbf{u}_{h}, \mathbf{v}_{h}, p_{h}\right)$ is the solution of the equations (5.12-5.13) with (f, $\left.\mathbf{g}\right)$ in (5.12) substituted by $\mathbf{P}_{h}(\operatorname{curl} \xi, \mathbf{0})$. In particular, by using $(\boldsymbol{\phi}, \boldsymbol{\psi})=\left(\mathbf{u}_{h}, \mathbf{v}_{h}\right)$ in (5.12) and $q=p_{h}$ in (5.13) 
we may write

$$
a\left(\left(\mathbf{u}_{h}, \mathbf{v}_{h}\right),\left(\mathbf{u}_{h}, \mathbf{v}_{h}\right)\right)+\frac{1}{\rho_{\mathrm{F}} c^{2}} \int_{\Omega_{\mathrm{F}}} p_{h}^{2}=d\left(\mathbf{P}_{h}(\operatorname{curl} \xi, \mathbf{0}),\left(\mathbf{u}_{h}, \mathbf{v}_{h}\right)\right) .
$$

On the other hand, since (5.13) implies that $\operatorname{div} u_{h}=\frac{1}{\rho_{\mathrm{F}} c^{2}} p_{h}$, we know that

$$
\left\|\left(\mathbf{u}_{h}, \mathbf{v}_{h}\right)\right\|^{2} \leq C\left[a\left(\left(\mathbf{u}_{h}, \mathbf{v}_{h}\right),\left(\mathbf{u}_{h}, \mathbf{v}_{h}\right)\right)+\frac{1}{\rho_{\mathrm{F}} c^{2}} \int_{\Omega_{\mathrm{F}}} p_{h}^{2}\right]
$$

even for $c=\infty$ because of Lemma 5.1 (i). All together, the last three equations show that

$$
\left\|\widetilde{\mathbf{T}}_{h}(\operatorname{curl} \xi, \mathbf{0})\right\|^{2} \leq C d\left(\mathbf{P}_{h}(\operatorname{curl} \xi, \mathbf{0}),\left(\mathbf{u}_{h}, \mathbf{v}_{h}\right)\right) .
$$

Now, since $\left(\mathbf{u}_{h}, \mathbf{v}_{h}\right) \in \mathbf{G}_{h} \subset \mathbf{K}_{h}^{\perp_{\mathrm{H}}}$ and $(\operatorname{curl} \xi, \mathbf{0}) \in \mathbf{K}$,

$$
\begin{aligned}
d\left(\mathbf{P}_{h}(\operatorname{curl} \xi, \mathbf{0}),\left(\mathbf{u}_{h}, \mathbf{v}_{h}\right)\right) & =d\left((\operatorname{curl} \xi, \mathbf{0}),\left(\mathbf{u}_{h}, \mathbf{v}_{h}\right)\right) \\
& =d\left((\operatorname{curl} \xi, \mathbf{0}),\left(\mathbf{u}_{h}, \mathbf{v}_{h}\right)-(\tilde{\mathbf{u}}, \tilde{\mathbf{v}})\right)
\end{aligned}
$$

for any $(\tilde{\mathbf{u}}, \tilde{\mathbf{v}}) \in \mathbf{K}^{\perp_{\mathrm{H}}}$. In particular, let us take $(\tilde{\mathbf{u}}, \tilde{\mathbf{v}}):=\left(\nabla \chi, \mathbf{v}_{h}\right)$, with $\chi \in H^{1}\left(\Omega_{\mathrm{F}}\right) / \mathcal{P}_{0}$ being the solution of the Neumann problem

$$
\begin{array}{ll}
\Delta \chi=\operatorname{div} \mathbf{u}_{h}, & \text { in } \Omega_{\mathrm{F}}, \\
\frac{\partial \chi}{\partial \boldsymbol{\nu}}=\mathbf{u}_{h} \cdot \boldsymbol{\nu}, & \text { on } \Gamma_{\mathrm{F}} .
\end{array}
$$

Hence, there exists $\zeta \in H^{1}\left(\Omega_{\mathrm{F}}\right)$ such that $(\tilde{\mathbf{u}}, \tilde{\mathbf{v}})-\left(\nabla \chi, \mathbf{v}_{h}\right)=(\operatorname{curl} \zeta, \mathbf{0}) \in \mathbf{K}$. On the other hand, because of the usual a priori estimate, $\chi \in H^{3 / 2}\left(\Omega_{\mathrm{F}}\right) / \mathcal{P}_{0}$ and

$$
\begin{aligned}
\|\nabla \chi\|_{H^{1 / 2}\left(\Omega_{\mathrm{F}}\right)} & \leq C\left[\left\|\operatorname{div} \mathbf{u}_{h}\right\|_{L^{2}\left(\Omega_{\mathrm{F}}\right)}+\left\|\mathbf{u}_{h} \cdot \boldsymbol{\nu}\right\|_{L^{2}\left(\Gamma_{\mathrm{F}}\right)}\right] \\
& \leq C\left\|\left(\mathbf{u}_{h}, \mathbf{v}_{h}\right)\right\| \leq C\left|\mathbf{P}_{h}(\operatorname{curl} \xi, \mathbf{0})\right| \leq C|(\mathbf{f}, \mathbf{g})|,
\end{aligned}
$$

where we have used (5.14) to bound $\left\|\left(\mathbf{u}_{h}, \mathbf{v}_{h}\right)\right\|$. Thus, the arguments in the proof of Lemma 5.5 of [3] can be repeated to prove that

$$
\left|\left(\mathbf{u}_{h}, \mathbf{v}_{h}\right)-(\tilde{\mathbf{u}}, \tilde{\mathbf{v}})\right|=\|\operatorname{curl} \zeta\|_{L^{2}\left(\Omega_{\mathrm{F}}\right)^{2}} \leq C h^{1 / 2}\|\nabla \chi\|_{H^{1 / 2}\left(\Omega_{\mathrm{F}}\right)} \leq C h^{1 / 2}|(\mathbf{f}, \mathbf{g})| .
$$

Therefore, (6.9), (6.10) and (6.11) yield

$$
\left\|\widetilde{\mathbf{T}}_{h}(\operatorname{curl} \xi, \mathbf{0})\right\|^{2} \leq C h^{1 / 2}|(\mathbf{f}, \mathbf{g})|^{2},
$$

which together with (6.7) and (6.8) allow us to conclude the lemma.

As a consequence of this lemma isolated parts of $\sigma(\widetilde{\mathbf{T}})$ are approximated by isolated parts of $\sigma\left(\widetilde{\mathbf{T}}_{h}\right)$ (see [14]). More precisely, for any eigenvalue $\lambda$ of $\widetilde{\mathbf{T}}$ of finite multiplicity $m$, there exist exactly $m$ eigenvalues $\lambda_{h}^{(1)} \ldots \lambda_{h}^{(m)}$ of $\widetilde{\mathbf{T}}_{h}$ (repeated according to their respective multiplicities) converging to $\lambda$ as $h$ goes to zero. Furthermore, no spurious modes can arise as it is typical with other discretizations of displacement formulations (see for instance [15]). We state this result in the following theorem.

Theorem 6.2. Let $I$ be a closed interval such that $I \cap \sigma(\mathbf{T})=\emptyset$. There exists a strictly positive constant $h_{I}$ such that if $h \leq h_{I}$ then $I \cap \sigma\left(\mathbf{T}_{h}\right)=\emptyset$.

From now on and until the end of this section, let $\lambda \in(0,1)$ be a fixed eigenvalue of $\mathbf{T}$ of finite multiplicity $m$ and let $\mathbf{E} \subset \mathbf{G}$ be its associated eigenspace. Then $\lambda$ is also an eigenvalue of $\widetilde{\mathbf{T}}$ with the same multiplicity and 
eigenspace. Let $\lambda_{h}^{(1)} \ldots \lambda_{h}^{(m)}$ be the $m$ eigenvalues of $\widetilde{\mathbf{T}}_{h}$ converging to $\lambda$ and let $\mathbf{E}_{h}$ be the direct sum of the corresponding eigenspaces. For $h$ small enough, all the $\lambda_{h}^{(i)}$ are strictly positive and hence they are also eigenvalues of $\mathbf{T}_{h}$ with the same associated eigenspaces; furthermore, $\mathbf{E}_{h} \subset \mathbf{G}_{h}$.

So, by applying the spectral approximation theory for compact operators as stated in [1] (Theorem 7.1) to $\widetilde{\mathbf{T}}_{h}$ and by using Lemma 6.3 , we have the following error estimates.

Theorem 6.3. There exist a strictly positive constant $C$, independent of $h$ and $c$, such that

i) for each $\left(\mathbf{u}_{h}, \mathbf{v}_{h}\right) \in \mathbf{E}_{h}$, dist $\left(\left(\mathbf{u}_{h}, \mathbf{v}_{h}\right), \mathbf{E}\right) \leq C h^{r}\left\|\left(\mathbf{u}_{h}, \mathbf{v}_{h}\right)\right\|$,

ii) for each $(\mathbf{u}, \mathbf{v}) \in \mathbf{E}$, dist $\left((\mathbf{u}, \mathbf{v}), \mathbf{E}_{h}\right) \leq C h^{r}\|(\mathbf{u}, \mathbf{v})\|$,

where dist denotes the distance in the norm $\|\cdot\|$ and $r:=\min \{s, t\}$, with $s \in\left[\frac{1}{2}, 1\right]$ and $t \in(0,1]$ as in Lemmas 4.2 and 4.3 , respectively.

Finally, regarding the eigenvalues, we prove the following theorem providing an optimal double order of convergence.

Theorem 6.4. There exist a strictly positive constant $C$, independent of $h$ and $c$, such that

$$
\left|\lambda-\lambda_{h}^{(i)}\right| \leq C h^{2 r}, \quad i=1, \ldots, m,
$$

where $r:=\min \{s, t\}$, with $s \in\left[\frac{1}{2}, 1\right]$ and $t \in(0,1]$ as in Lemmas 4.2 and 4.3, respectively.

Proof. It is easy to check that $\widetilde{\mathbf{T}}$ and $\widetilde{\mathbf{T}}_{h}$ are selfadjoint in $\mathbf{H}$ with respect to the inner product $d$. Since by Lemma $6.4 \lim _{h \rightarrow 0}\left|\widetilde{\mathbf{T}}-\widetilde{\mathbf{T}}_{h}\right|=0$, then by specializing Theorem 7.3 of [1] to these operators we have that

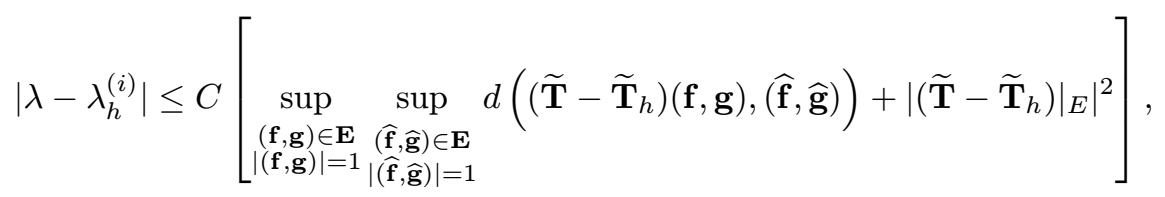

for $i=1, \cdots, m$. Because of Lemma 6.3 we just need to estimate the first term in the right hand side of this expression.

Let $(\mathbf{f}, \mathbf{g})$ and $(\widehat{\mathbf{f}}, \widehat{\mathbf{g}})$ in $\mathbf{E}$ and let

$$
\begin{aligned}
(\mathbf{u}, \mathbf{v}) & :=\widetilde{\mathbf{T}}(\mathbf{f}, \mathbf{g}) \in \mathbf{G}, & (\widehat{\mathbf{u}}, \widehat{\mathbf{v}}) & :=\widetilde{\mathbf{T}}(\widehat{\mathbf{f}}, \widehat{\mathbf{g}}) \in \mathbf{G} \\
\left(\mathbf{u}_{h}, \mathbf{v}_{h}\right) & :=\widetilde{\mathbf{T}}_{h}(\mathbf{f}, \mathbf{g}) \in \mathbf{G}_{h}, & \left(\widehat{\mathbf{u}}_{h}, \widehat{\mathbf{v}}_{h}\right) & :=\widetilde{\mathbf{T}}_{h}(\widehat{\mathbf{f}}, \widehat{\mathbf{g}}) \in \mathbf{G}_{h} .
\end{aligned}
$$

Furthermore, let $p, \widehat{p} \in Q$ and $p_{h}, \widehat{p}_{h} \in Q_{h}$ be the corresponding pressures associated by equations (3.9-3.10) and (5.12)-(5.13), respectively. For the sake of notational simplicity we denote:

$$
\begin{gathered}
U:=(\mathbf{u}, \mathbf{v}, p), \quad \widehat{U}:=(\widehat{\mathbf{u}}, \widehat{\mathbf{v}}, \widehat{p}), \quad U_{h}:=\left(\mathbf{u}_{h}, \mathbf{v}_{h}, p_{h}\right), \quad \widehat{U}_{h}:=\left(\widehat{\mathbf{u}}_{h}, \widehat{\mathbf{v}}_{h}, \widehat{p}_{h}\right), \\
A(U, \widehat{U}):=a((\mathbf{u}, \mathbf{v}),(\widehat{\mathbf{u}}, \widehat{\mathbf{v}}))+b(p,(\widehat{\mathbf{u}}, \widehat{\mathbf{v}}))+b(\widehat{p},(\mathbf{u}, \mathbf{v}))-\frac{1}{\rho_{\mathrm{F}} c^{2}} \int_{\Omega_{\mathrm{F}}} p \widehat{p} \\
F:=(\mathbf{f}, \mathbf{g}, 0), \quad \widehat{F}:=(\widehat{\mathbf{f}}, \widehat{\mathbf{g}}, 0), \quad D(U, \widehat{F}):=d((\mathbf{u}, \mathbf{v}),(\widehat{\mathbf{f}}, \widehat{\mathbf{g}})) .
\end{gathered}
$$

So, we have to bound $d\left(\left(\widetilde{\mathbf{T}}-\widetilde{\mathbf{T}}_{h}\right)(\mathbf{f}, \mathbf{g}),(\widehat{\mathbf{f}}, \widehat{\mathbf{g}})\right)=D\left(U-U_{h}, \widehat{F}\right)$. 
It is simple to check that

$$
\begin{aligned}
D\left(U-U_{h}, \widehat{F}\right) & =A(U, \widehat{U})-A\left(U_{h}, \widehat{U}_{h}\right) \\
& =A\left(U-U_{h}, \widehat{U}-\widehat{U}_{h}\right)+A\left(U-U_{h}, \widehat{U}_{h}\right)-A\left(U_{h}, \widehat{U}-\widehat{U}_{h}\right) \\
& =A\left(U-U_{h}, \widehat{U}-\widehat{U}_{h}\right)+\left[A\left(U, \widehat{U}_{h}\right)-D\left(F, \widehat{U}_{h}\right)\right]+\left[A\left(U_{h}, \widehat{U}\right)-D\left(U_{h}, \widehat{F}\right)\right] .
\end{aligned}
$$

Since $(\mathbf{f}, \mathbf{g})$ and $(\widehat{\mathbf{f}}, \widehat{\mathbf{g}})$ are eigenfunctions of $\mathbf{T}$ belonging to $\mathbf{K}^{\perp_{\mathrm{H}}}$, the first term in the right hand side of this equation is easily bounded by using Lemma 6.2 and the estimate (6.6) for the pressure:

$$
\left|A\left(U-U_{h}, \widehat{U}-\widehat{U}_{h}\right)\right| \leq C h^{2 r}|(\mathbf{f}, \mathbf{g})||(\widehat{\mathbf{f}}, \widehat{\mathbf{g}})| .
$$

The other two consistency terms are due to the nonconformity of the method and both can be handled identically because of the symmetry of $A$ and $D$. For instance, for the first one we have:

$$
\left[A\left(U, \widehat{U}_{h}\right)-D\left(F, \widehat{U}_{h}\right)\right]=a\left((\mathbf{u}, \mathbf{v}),\left(\widehat{\mathbf{u}}_{h}, \widehat{\mathbf{v}}_{h}\right)\right)+b\left(p,\left(\widehat{\mathbf{u}}_{h}, \widehat{\mathbf{v}}_{h}\right)\right)-d\left((\mathbf{f}, \mathbf{g}),\left(\widehat{\mathbf{u}}_{h}, \widehat{\mathbf{v}}_{h}\right)\right)
$$

This expression coincides with the left hand side of (6.5) and can be dealt with in the same way to obtain:

$$
a\left((\mathbf{u}, \mathbf{v}),\left(\widehat{\mathbf{u}}_{h}, \widehat{\mathbf{v}}_{h}\right)\right)+b\left(p,\left(\widehat{\mathbf{u}}_{h}, \widehat{\mathbf{v}}_{h}\right)\right)-d\left((\mathbf{f}, \mathbf{g}),\left(\widehat{\mathbf{u}}_{h}, \widehat{\mathbf{v}}_{h}\right)\right)=\int_{\Gamma_{\mathrm{I}}} p\left(\widehat{\mathbf{u}}_{h} \cdot \boldsymbol{\nu}-\widehat{\mathbf{v}}_{h} \cdot \boldsymbol{\nu}\right) d \Gamma
$$

however, since $\left(\widehat{\mathbf{u}}_{h}, \widehat{\mathbf{v}}_{h}\right)=\widetilde{\mathbf{T}}_{h}(\widehat{\mathbf{f}}, \widehat{\mathbf{g}})$, the estimate $(6.5)$ can be improved by proceeding as in [18]. In fact, let $P_{h}$ denote the $L^{2}\left(\Gamma_{\mathrm{I}}\right)$-projection onto the piecewise constant functions. Since $\left(\widehat{\mathbf{u}}_{h}, \widehat{\mathbf{v}}_{h}\right) \in \mathbf{V}_{h}$, the discrete interface condition implies that $\widehat{\mathbf{u}}_{h} \cdot \boldsymbol{\nu}=P_{h}\left(\widehat{\mathbf{v}}_{h} \cdot \boldsymbol{\nu}\right)$ and hence

$$
\begin{aligned}
\left|\int_{\Gamma_{\mathrm{I}}} p\left(\widehat{\mathbf{u}}_{h} \cdot \boldsymbol{\nu}-\widehat{\mathbf{v}}_{h} \cdot \boldsymbol{\nu}\right) d \Gamma\right| & =\left|\int_{\Gamma_{\mathrm{I}}}\left[p-P_{h}(p)\right]\left[P_{h}\left(\widehat{\mathbf{v}}_{h} \cdot \boldsymbol{\nu}\right)-\widehat{\mathbf{v}}_{h} \cdot \boldsymbol{\nu}\right] d \Gamma\right| \\
& \leq\left\|p-P_{h}(p)\right\|_{L^{2}\left(\Gamma_{\mathrm{I}}\right)}\left\|P_{h}\left(\widehat{\mathbf{v}}_{h} \cdot \boldsymbol{\nu}\right)-\widehat{\mathbf{v}}_{h} \cdot \boldsymbol{\nu}\right\|_{L^{2}\left(\Gamma_{\mathrm{I}}\right)} .
\end{aligned}
$$

Since $(\mathbf{f}, \mathbf{g}) \in \mathbf{E}$, according to Theorem 4.3, $p \in H^{1+s}\left(\Omega_{\mathrm{F}}\right)$, with $s \geq 1 / 2$, and $\|p\|_{H^{1+s}\left(\Omega_{\mathrm{F}}\right)} \leq C|(\mathbf{f}, \mathbf{g})| ;$ hence, it holds

$$
\left\|p-P_{h}(p)\right\|_{L^{2}\left(\Gamma_{\mathrm{I}}\right)} \leq C h\|p\|_{H^{1}\left(\Gamma_{\mathrm{I}}\right)} \leq C h\|p\|_{H^{3 / 2}\left(\Omega_{\mathrm{F}}\right)} \leq C h|(\mathbf{f}, \mathbf{g})|
$$

On the other hand,

$$
\left\|P_{h}\left(\widehat{\mathbf{v}}_{h} \cdot \boldsymbol{\nu}\right)-\widehat{\mathbf{v}}_{h} \cdot \boldsymbol{\nu}\right\|_{L^{2}\left(\Gamma_{\mathrm{I}}\right)} \leq\left\|\left(I-P_{h}\right)\left(\widehat{\mathbf{v}} \cdot \boldsymbol{\nu}-\widehat{\mathbf{v}}_{h} \cdot \boldsymbol{\nu}\right)\right\|_{L^{2}\left(\Gamma_{\mathrm{I}}\right)}+\left\|\left(I-P_{h}\right)(\widehat{\mathbf{v}} \cdot \boldsymbol{\nu})\right\|_{L^{2}\left(\Gamma_{\mathrm{I}}\right)} .
$$

The first term in the right hand side of (6.15) is easily bounded by using Lemma 6.3:

$$
\begin{aligned}
\left\|\left(I-P_{h}\right)\left(\widehat{\mathbf{v}} \cdot \boldsymbol{\nu}-\widehat{\mathbf{v}}_{h} \cdot \boldsymbol{\nu}\right)\right\|_{L^{2}\left(\Gamma_{\mathrm{I}}\right)} & \leq\left\|\widehat{\mathbf{v}} \cdot \boldsymbol{\nu}-\widehat{\mathbf{v}}_{h} \cdot \boldsymbol{\nu}\right\|_{L^{2}\left(\Gamma_{\mathrm{I}}\right)} \\
& \leq C\left\|\widehat{\mathbf{v}}-\widehat{\mathbf{v}}_{h}\right\|_{H^{1}\left(\Omega_{\mathrm{S}}\right)^{2}} \leq C h^{r}|(\widehat{\mathbf{f}}, \widehat{\mathbf{g}})| .
\end{aligned}
$$

For the second term notice that, because of Theorem $4.3, \widehat{\mathbf{v}} \in H^{1+t}\left(\Omega_{\mathrm{S}}\right)^{2}$, with $t>0$, and $\|\widehat{\mathbf{v}}\|_{H^{1+t}\left(\Omega_{\mathrm{S}}\right)^{2}} \leq$ $C|(\widehat{\mathbf{f}}, \widehat{\mathbf{g}})|$; hence, for $\tilde{r}:=\min \{1, t+1 / 2\}$, it holds

$$
\begin{aligned}
\left\|\left(I-P_{h}\right)(\widehat{\mathbf{v}} \cdot \boldsymbol{\nu})\right\|_{L^{2}\left(\Gamma_{\mathrm{I}}\right)} & \leq C h^{\tilde{r}}\|\widehat{\mathbf{v}} \cdot \boldsymbol{\nu}\|_{H^{\tilde{r}}\left(\Gamma_{\mathrm{I}}\right)} \leq C h^{\tilde{r}}\|\widehat{\mathbf{v}}\|_{H^{1 / 2+\tilde{r}}\left(\Omega_{\mathrm{S}}\right)^{2}} \\
& \leq C h^{\tilde{r}}\|\widehat{\mathbf{v}}\|_{H^{1+t}\left(\Omega_{\mathrm{S}}\right)^{2}} \leq C h^{\tilde{r}}|(\widehat{\mathbf{f}}, \widehat{\mathbf{g}})| .
\end{aligned}
$$


Finally, since $r \leq \tilde{r},(6.13-6.17)$ yield

$$
\left|\int_{\Gamma_{\mathrm{I}}} p\left(\widehat{\mathbf{u}}_{h} \cdot \boldsymbol{\nu}-\widehat{\mathbf{v}}_{h} \cdot \boldsymbol{\nu}\right) d \Gamma\right| \leq C h^{1+r}|(\mathbf{f}, \mathbf{g})||(\widehat{\mathbf{f}}, \widehat{\mathbf{g}})| \leq C h^{2 r}|(\mathbf{f}, \mathbf{g})||(\widehat{\mathbf{f}}, \widehat{\mathbf{g}})|,
$$

which allows us to conclude the theorem.

\section{NUMERICAL EXPERIMENTS}

We have applied the method analyzed above to the computation of the vibration modes of a 2D steel vessel partially filled with water as that in Figure 1. We have used the following physical parameters for the steel: density $\rho_{\mathrm{S}}=7.7 \times 10^{3} \mathrm{~kg} / \mathrm{m}^{3}$, Young modulus $E=1.44 \times 10^{11} \mathrm{~Pa}$ and Poisson ratio $\nu_{\mathrm{S}}=0.35$ (the Lamé coefficients being $\lambda_{\mathrm{S}}=\frac{E \nu_{\mathrm{S}}}{\left(1+\nu_{\mathrm{S}}\right)\left(1-2 \nu_{\mathrm{S}}\right)}$ and $\left.\mu_{\mathrm{S}}=\frac{E}{1+\nu_{\mathrm{S}}}\right)$. The water has been idealized as perfectly incompressible $(c=\infty)$ and with density $\rho_{\mathrm{F}}=10^{3} \mathrm{~kg} / \mathrm{m}^{3}$.

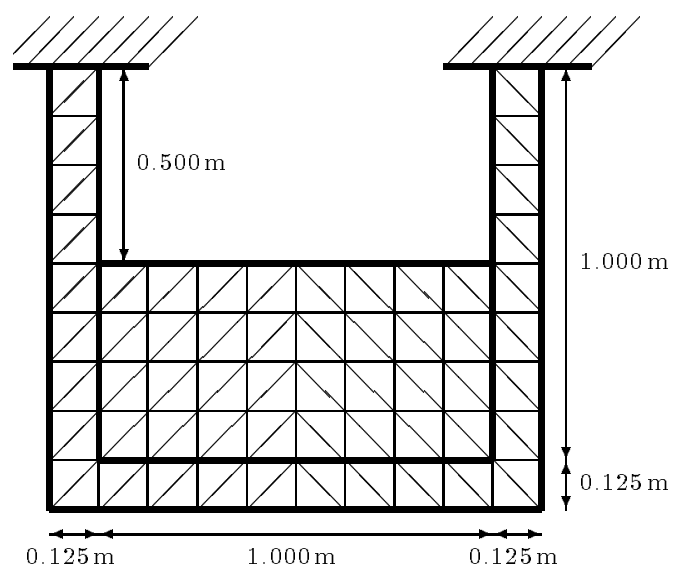

FiguRE 2. Steel vessel filled with water: geometrical data and initial mesh.

Figure 2 shows the geometrical data of the fluid and solid domains and the coarsest used mesh. To estimate the order of convergence of the method, the vibration modes of this coupled problem have been computed using several uniform refinements of this mesh. The refinement parameter $N$ denotes the number of layers of triangles in the solid; the mesh in Figure 2 corresponds to $N=1$.

The eigenvalue problem to be solved is the discrete version of problem (3.4-3.5) for $c=\infty$ :

Find $\lambda_{h} \in \mathbb{R},\left(\mathbf{u}_{h}, \mathbf{v}_{h}, p_{h}\right) \in \mathbf{V}_{h} \times Q_{h},\left(\mathbf{u}_{h}, \mathbf{v}_{h}, p_{h}\right) \neq(\mathbf{0}, \mathbf{0}, 0)$, such that:

$$
\begin{gathered}
\int_{\Omega_{\mathrm{S}}} \boldsymbol{\sigma}\left(\mathbf{v}_{h}\right): \boldsymbol{\varepsilon}(\boldsymbol{\psi})+\int_{\Gamma_{\mathrm{O}}} \rho_{\mathrm{F}} g \mathbf{u}_{h} \cdot \boldsymbol{\nu} \boldsymbol{\phi} \cdot \boldsymbol{\nu} d \Gamma+\int_{\Gamma_{\mathrm{I}}} \rho_{\mathrm{F}} g \mathbf{k} \cdot \boldsymbol{\nu} \mathbf{v}_{h} \cdot \boldsymbol{\nu} \boldsymbol{\psi} \cdot \boldsymbol{\nu} d \Gamma-\int_{\Omega_{\mathrm{F}}} p_{h} \operatorname{div} \boldsymbol{\phi}= \\
\lambda_{h}\left(\int_{\Omega_{\mathrm{F}}} \rho_{\mathrm{F}} \mathbf{u}_{h} \cdot \boldsymbol{\phi}+\int_{\Omega_{\mathrm{S}}} \rho_{\mathrm{S}} \mathbf{v}_{h} \cdot \boldsymbol{\psi}\right), \quad \forall(\boldsymbol{\phi}, \boldsymbol{\psi}) \in \mathbf{V}_{h}, \\
\int_{\Omega_{\mathrm{F}}} q \operatorname{div} \mathbf{u}_{h}=0, \quad \forall q \in Q_{h} .
\end{gathered}
$$


TABLE 1. Computed eigenfrequencies (in rad/s) of the first sloshing modes.

\begin{tabular}{ccccc}
\hline Mode & $N=2$ & $N=3$ & $N=4$ & Order \\
\hline$\omega_{1}^{S}$ & 5.2946 & 5.3052 & 5.3090 & 1.99 \\
$\omega_{2}^{S}$ & 7.7334 & 7.7877 & 7.8071 & 1.94 \\
$\omega_{3}^{S}$ & 9.3491 & 9.4898 & 9.5414 & 1.87 \\
\hline
\end{tabular}

Since $\operatorname{div} \mathbf{u}_{h} \in Q_{h}$, equation (7.2) implies that $\operatorname{div} \mathbf{u}_{h}=0$ in $\Omega_{\mathrm{F}}$. Hence, in view of the discrete inf-sup condition of Lemma 5.1, the eigenvalue problem (7.1-7.2) is equivalent to the following one:

Find $\lambda_{h} \in \mathbb{R},\left(\mathbf{u}_{h}, \mathbf{v}_{h}\right) \in \mathbf{W}_{h},\left(\mathbf{u}_{h}, \mathbf{v}_{h}\right) \neq(\mathbf{0}, \mathbf{0})$, such that:

$$
\begin{aligned}
\int_{\Omega_{\mathrm{S}}} \boldsymbol{\sigma}\left(\mathbf{v}_{h}\right): \boldsymbol{\varepsilon}(\boldsymbol{\psi})+\int_{\Gamma_{\mathrm{O}}} \rho_{\mathrm{F}} g \mathbf{u}_{h} \cdot \boldsymbol{\nu} \boldsymbol{\phi} \cdot \boldsymbol{\nu} d \Gamma+\int_{\Gamma_{\mathrm{I}}} \rho_{\mathrm{F}} g \mathbf{k} \cdot \boldsymbol{\nu} \mathbf{v}_{h} \cdot \boldsymbol{\nu} \boldsymbol{\psi} \cdot \boldsymbol{\nu} d \Gamma= \\
\lambda_{h}\left(\int_{\Omega_{\mathrm{F}}} \rho_{\mathrm{F}} \mathbf{u}_{h} \cdot \boldsymbol{\phi}+\int_{\Omega_{\mathrm{S}}} \rho_{\mathrm{S}} \mathbf{v}_{h} \cdot \boldsymbol{\psi}\right), \quad \forall(\boldsymbol{\phi}, \boldsymbol{\psi}) \in \mathbf{W}_{h},
\end{aligned}
$$

where $\mathbf{W}_{h}=\left\{(\mathbf{u}, \mathbf{v}) \in \mathbf{V}_{h}: \operatorname{div} \mathbf{u}=0\right\}$, as defined in Section 5 above.

Now, since $\left\{\mathbf{u} \in \mathbf{R}_{h}\left(\Omega_{\mathrm{F}}\right): \operatorname{div} \mathbf{u}=0\right\}=\left\{\operatorname{curl} \xi: \xi \in L_{h}\left(\Omega_{\mathrm{F}}\right)\right\}$, problem (7.3) can be seen as a piecewise linear continuous discretization of a stream function formulation of the original problem. The interface condition reads $\int_{\ell} \frac{\partial \xi}{\partial \boldsymbol{\tau}} d \Gamma=\int_{\ell} \mathbf{v} \cdot \nu d \Gamma$ for each edge $\ell \subset \Gamma_{\mathrm{I}}$, where $\frac{\partial \xi}{\partial \boldsymbol{\tau}}$ stands for the tangential derivative of $\xi$, and we impose it by means of a Lagrange multiplier (see [4] for details).

Two kind of eigenmodes can be found by solving the eigenvalue problem (7.3):

- low frequency sloshing modes,

- hydroelastic vibration modes.

The first ones correspond to the gravity waves on the surface of the liquid, whereas the second ones are the vibration modes of the elastic vessel modified by the interaction with the liquid.

In Table 1 we show the frequencies $\omega_{i}^{S}$ of the three first sloshing modes (i.e., those corresponding to the three lowest eigenfrequecies) computed with different meshes. When the container is not rigid no exact solution is known for this problem, but the orders of convergence for each eigenfrequency (in powers of $h$ ) have been estimated by extrapolation; they are included in the last column of the table.

For a polygonal convex fluid domain as that in Figure 2, if the container were perfectly rigid, the order of convergence would be exactly 2 . Since for such low range of frequencies steel is almost rigid, the estimated orders are also very close to 2 . The rigidity of the steel for these sloshing modes can be observed in Figure 3 where the corresponding computed fluid displacement fields are shown: it can be seen that their normal components are practically null on the whole fluid-solid interface.

In Table 2 we show the computed frequencies $\omega_{i}^{H}$ of the four lowest frequency hydroelastic vibration modes. Once more no exact solution is known and the orders of convergence included in the table have been estimated by extrapolation.

The orders of convergence are now significantly smaller than those of the sloshing modes. This is due to the fact that the displacement fields corresponding to the vibration modes of the vessel have strong singularities at the non convex angles of the solid domain. In fact, according to [13], constant $t$ in Lemma 4.3 is approximately 0.68 for our problem. Hence, the order of convergence in that case should be $2 t \approx 1.36$.

Figures 4 to 7 show the deformed structure and the fluid displacement and pressure fields for these hydroelastic vibration modes. 
FINITE ELEMENT ANALYSIS OF SLOSHING AND HYDROELASTIC VIBRATIONS UNDER GRAVITY

Eigenfrequency: $\omega_{1}^{S}$

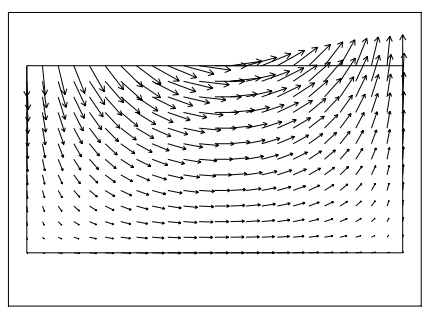

Eigenfrequency: $\omega_{2}^{S}$

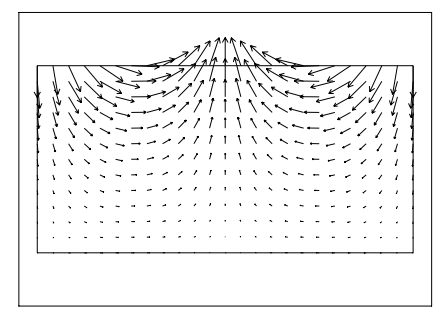

Eigenfrequency: $\omega_{3}^{S}$

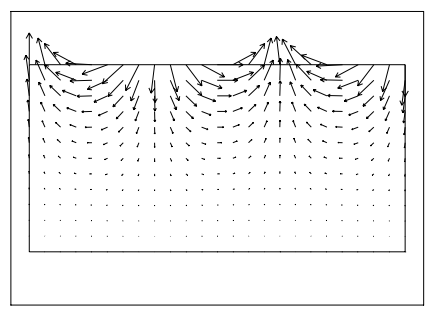

Figure 3. Fluid displacement fields of the lowest frequency sloshing modes.

TABLE 2. Computed eigenfrequencies (in $\mathrm{rad} / \mathrm{s}$ ) of the first hydroelastic vibration modes.

\begin{tabular}{ccccc}
\hline Mode & $N=2$ & $N=3$ & $N=4$ & Order \\
\hline$\omega_{1}^{H}$ & 627.8907 & 540.0423 & 502.7057 & 1.46 \\
$\omega_{2}^{H}$ & 2014.4331 & 1765.7687 & 1656.1781 & 1.36 \\
$\omega_{3}^{H}$ & 3487.2386 & 3145.0289 & 2978.5786 & 1.08 \\
$\omega_{4}^{H}$ & 3803.7429 & 3317.3062 & 3104.0943 & 1.37 \\
\hline
\end{tabular}

Fluid displacement field.

Deformed structure.
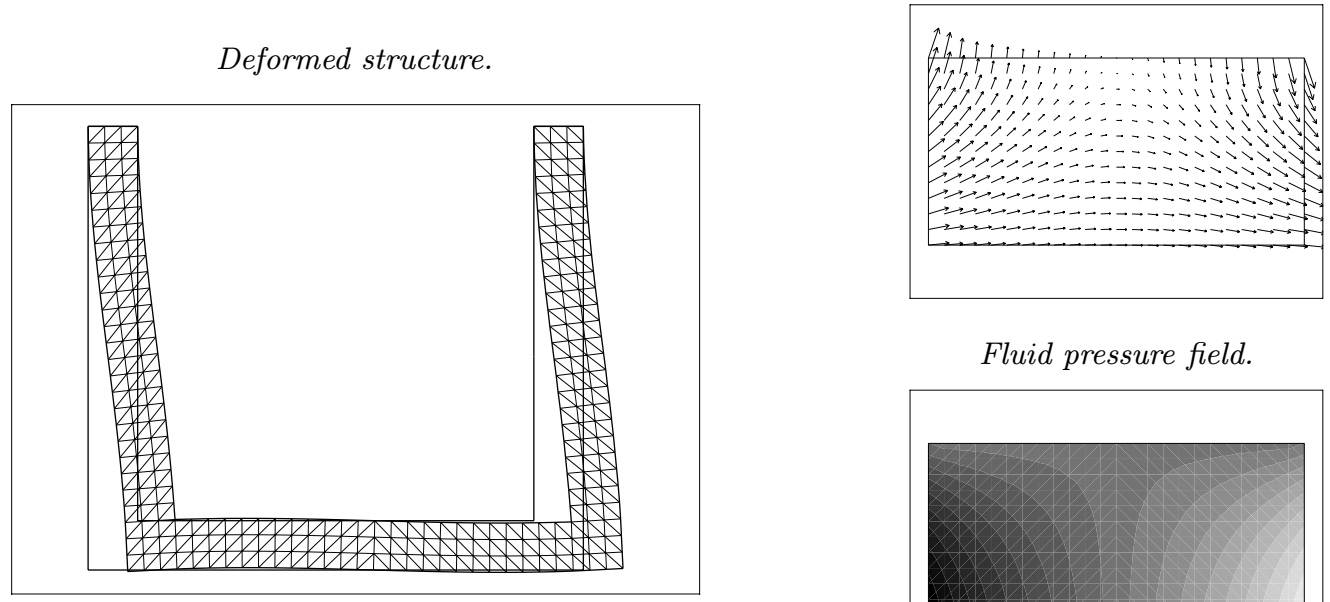

Fluid pressure field.

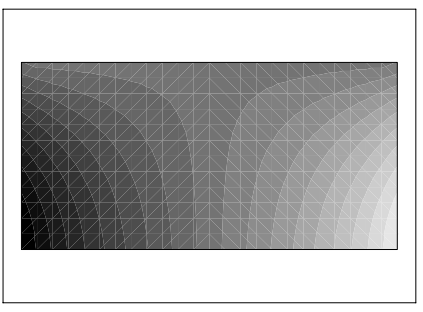

FigurE 4. Hydroelastic vibration mode of frequency $\omega_{1}^{H}$.

\section{Conclusions}

We have introduced a finite element method able to solve hydroelasticity vibration problems without neglecting gravity effects. We have proved convergence and given optimal error estimates for eigenmodes and eigenfrequencies. We have also proved that the method does not present spurious modes. The method can be used for incompressible as well as nearly incompressible fluids, and the obtained error estimates are independent of the acoustic speed. 
Fluid displacement field.

Deformed structure.
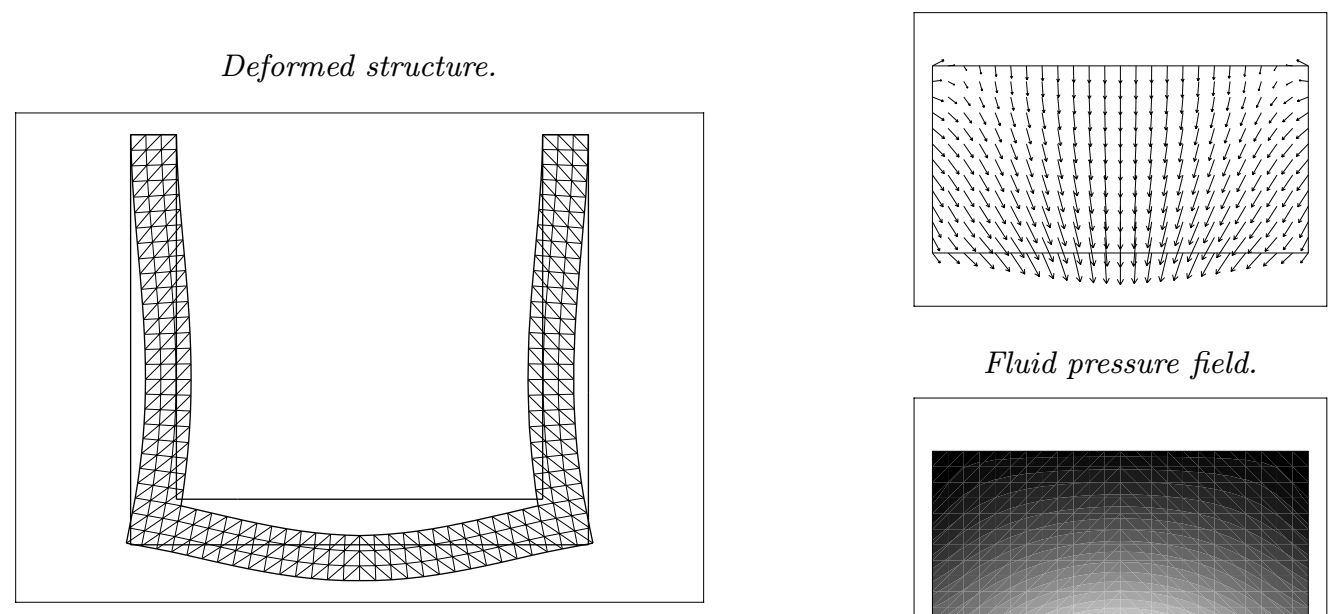

Fluid pressure field.

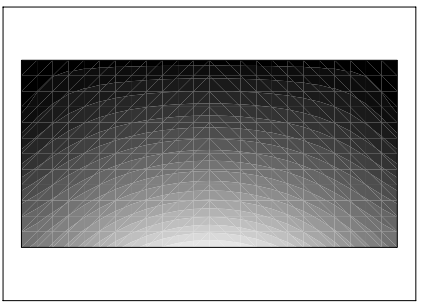

FiguRE 5. Hydroelastic vibration mode of frequency $\omega_{2}^{H}$.

Fluid displacement field.

Deformed structure.
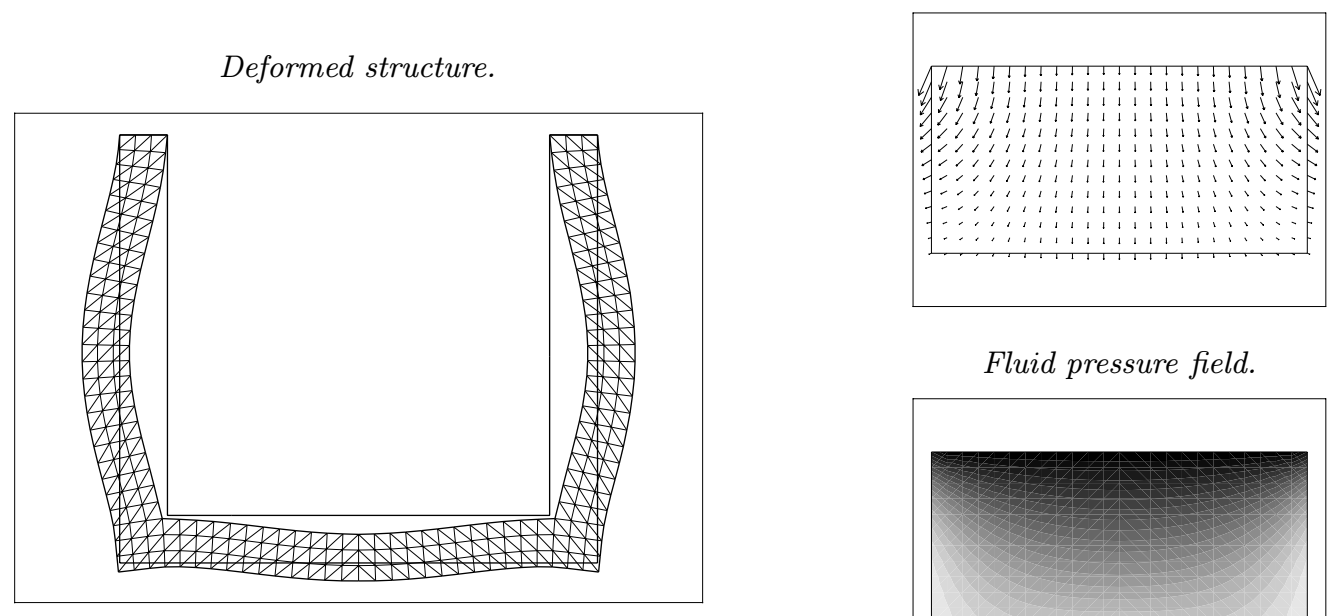

Fluid pressure field.

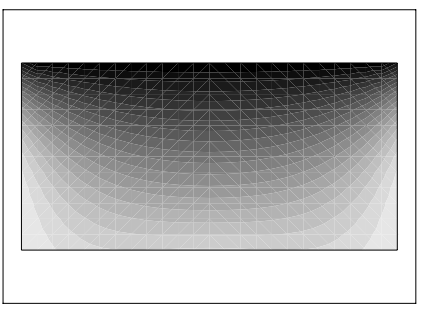

FiguRE 6. Hydroelastic vibration mode of frequency $\omega_{3}^{H}$.

The numerical results are in good agreement with the theoretical predictions for both, sloshing and elastic modes. Furthermore, the method still converges when the fluid domain has large reentrant interior angles (even of $2 \pi$ ). Therefore it provides a tool to simulate the performance of thin baffle-plates in reducing sloshing in liquid containers. The application of the method to this problem is to date under investigation.

The authors wish to thank Professor R. Ohayon for useful comments and discussions. 
Fluid displacement field.

Deformed structure.
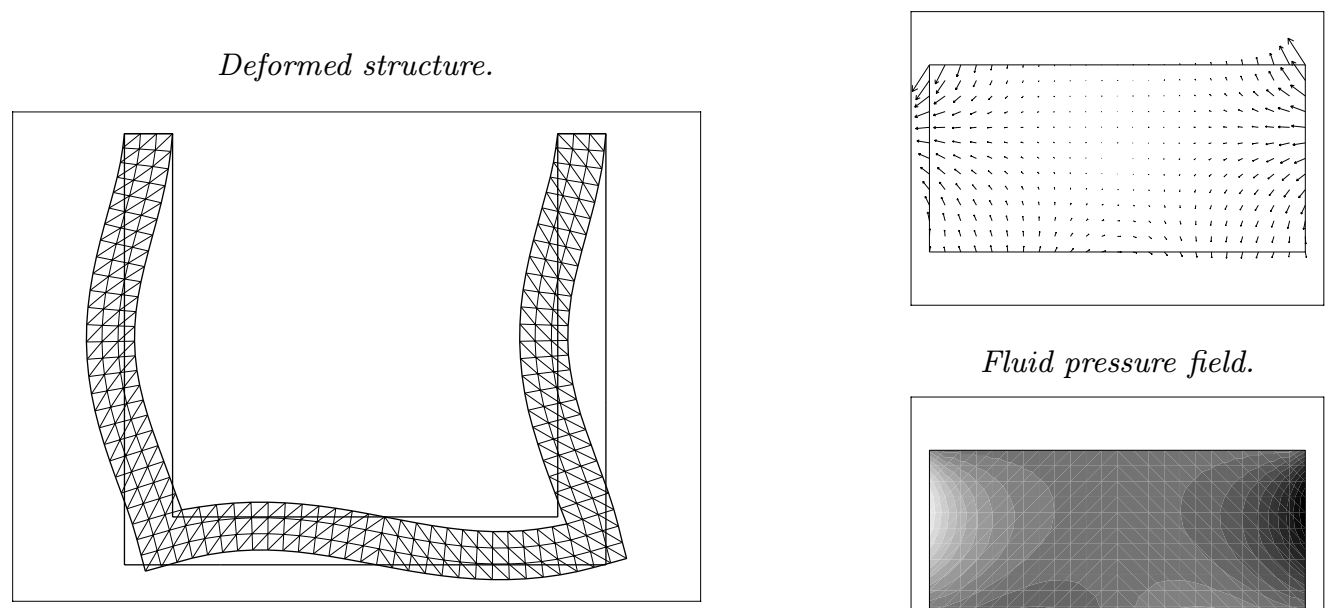

Fluid pressure field.

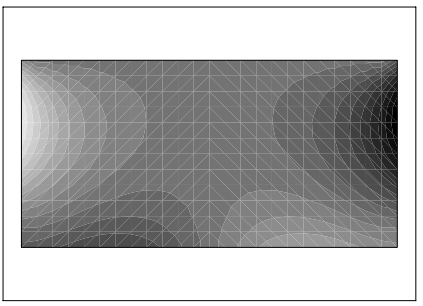

FigURE 7. Hydroelastic vibration mode of frequency $\omega_{4}^{H}$.

\section{REFERENCES}

[1] I. Babuška and J. Osborn, Eigenvalue problems, in Handbook of Numerical Analysis, Vol. II, P.G. Ciarlet and J.L. Lions Eds., North Holland, Amsterdam (1991).

[2] H. Berger, J. Boujot and R. Ohayon, On a spectral problem in vibration mechanics: computation of elastic tanks partially filled with liquids. J. Math. Anal. Appl. 51 (1975) 272-298.

[3] A. Bermúdez, R. Durán, M.A. Muschietti, R. Rodríguez and J. Solomin, Finite element vibration analysis of fluid-solid systems without spurious modes. SIAM J. Numer. Anal. 32 (1995) 1280-1295.

[4] A. Bermúdez, R. Durán and R. Rodríguez, Finite element analysis of compressible and incompressible fluid-solid systems. Math. Comp. 67 (1998) 111-136.

[5] A. Bermúdez and R. Rodríguez, Finite element computation of the vibration modes of a fluid-solid system. Comp. Methods Appl. Mech. Eng. 119 (1994) 355-370.

[6] J. Boujot, Sur l'analyse des caractéristiques vibratoirs d'un liquide contenu dans un reservoir. J. Mécanique 11 (1972) 647-671.

[7] J. Boujot, Mathematical formulation of fluid-structure interaction problems. RAIRO Modél. Math. Anal. Numér. 21 (1987) 239-260.

[8] F. Brezzi and M. Fortin, Mixed and Hybrid Finite Element Methods. Springer-Verlag, New York (1991).

[9] P. Clement, Approximation by finite element functions using local regularization. RAIRO Anal. Numér. 9 (1975) 77-84.

[10] C. Conca, J. Planchard and M. Vanninathan, Fluids and Periodic Structures. Masson, Paris (1992).

[11] J. Descloux, N. Nassif and J. Rappaz, On spectral approximation. Part I: The problem of convergence. Part 2: Error estimates for the Galerkin methods. RAIRO Anal. Numér. 12 (1978) 97-119.

[12] V. Girault and P.A. Raviart, Finite Element Methods for Navier-Stokes Equations. Springer-Verlag, Berlin, Heidelberg, New York, Tokyo (1986).

[13] P. Grisvard, Elliptic Problems for Non-smooth Domains. Pitman, Boston (1985).

[14] T. Kato, Perturbation Theory for Linear Operators. Springer Verlag, Berlin (1966).

[15] M. Hamdi, Y. Ouset and G. Verchery, A displacement method for the analysis of vibrations of coupled fluid-structure systems, Internat. J. Numer. Methods Eng. 13 (1978) 139-150.

[16] J. Mandel, Cours de Mécanique des Milieux Continus. Gauthier-Villars, Paris (1966).

[17] H.J-P. Morand and R. Ohayon, Interactions Fluides-Structures, Recherches en Mathématiques Appliquées. Masson, Paris 23 (1992).

[18] R. Rodríguez and J. Solomin, The order of convergence of eigenfrequencies in finite element approximations of fluid-structure interaction problems. Math. Comp. 65 (1996) 1463-1475.

[19] R.M.S.M. Schulkes, Interactions of an elastic solid with a viscous fluid: eigenmode analysis. J. Comput. Phys. 100 (1992) 270-283.

[20] P.A. Raviart and J.M. Thomas, A mixed finite element method for second order elliptic problems, in Mathematical Aspects of Finite Element Methods, Lecture Notes in Mathematics. Springer Verlag, Berlin, Heidelberg, New York 606 (1977) $292-315$.

[21] P. Tong, Liquid sloshing in an elastic container. AFOSR 66-0943, California Institute of Technology, Pasadena, CA (1966). 